\title{
Cost-sharing in Directed Networks: Experimental Study of Equilibrium Choice and System Dynamics
}

\author{
Caiyun Liu \\ Northwestern University \\ Kellogg School of Management, 2001 Sheridan Road \\ Evanston, Il 60208 \\ Email: c-liu@kellogg.northwestern.edu \\ Vincent Mak \\ University of Cambridge \\ Cambridge Judge Business School, Trumpington Street \\ Cambridge CB2 1AG, United Kingdom \\ Email: v.mak@jbs.cam.ac.uk \\ Amnon Rapoport* \\ University of California, Riverside \\ A. Gary Anderson Graduate School of Management \\ 900 University Ave., Riverside CA, 92521 \\ Email: amnonr@ucr.edu
}

Forthcoming in Journal of Operations Management (Special Issue on System Dynamics)

* Corresponding author

Keywords: cost-sharing, system dynamics, positive externalities, experiment

Acknowledgement: This research was supported by NSF Grant SES-1418923 awarded to the University of Nevada, Las Vegas. 


\title{
Cost-sharing in Directed Networks: Experimental Study of Equilibrium Choice and System Dynamics
}

\begin{abstract}
This study reports the results of an experiment on directed networks with positive externalities induced by cost-sharing. Subjects participated in a network game in which they had to choose between private and public transportations. If a player chose public transportation, then she shared the travel cost equally with other players making the same choice, whereas if she chose private transportation, then her travel cost was fixed. Travel costs on the private route were manipulated across the two experimental conditions. In one condition, these costs were homogeneous among players; in the other condition, they were heterogeneous among players and only privately known. We found that half (none) of the player groups in the homogeneous (heterogeneous) condition converged towards the efficient equilibrium. Examination of the system dynamics shows that convergence towards efficiency was facilitated by: (1) the existence of an intermediate equilibrium choice; and (2) strategic teaching by which a farsighted player chooses strategies with poor short-term payoff in order to shift group decisions to the efficient equilibrium and thereby increase her own long-term benefit.
\end{abstract}




\section{Introduction}

Transportation networks provide the foundation for the movement of people and goods across space and time, and are essential to the functioning of modern societies. The design and control of such networks requires thorough understanding of fundamental physical, behavioral, and social issues that are major research topics in the management, transportation research, economics, and computer sciences. Focusing on cost-sharing in directed networks, our study is positioned at the intersection of these disciplines.

In many settings in transportation networks, the overall behavior of the system is a complex product of the actions of multiple independent agents (e.g. drivers, commuters ...) who can generally be labelled as network users. These agents typically attempt to optimize their objective functions with no regard for the welfare of others. The externalities resulting from the decisions of each user are negative if individual benefits are a decreasing function of the number of other group members making similar choices. Examples include choice of routes in congestible networks (Cominetti, Correa, \& Stier-Moses 2006, 2009; Correa \& Stier-Moses 2011; Rapoport et al. 2009; Rapoport, Gisches, \& Mak 2014; Mak, Gisches, \& Rapoport 2014), and choice of time of departure in directed traffic networks with multiple bottlenecks (Daniel, Gisches, \& Rapoport 2009). In many other settings, the externalities are positive, as when the benefit of a choice of route is an increasing function of the number of other users making the same choice. This could happen, for example, when users of the same mode of transportation share the travel cost, as could happen with carpool and shuttle taxi. Previous experimental research on interactive decision behavior in networks (e.g., Daniel et al. 2009; Gisches \& Rapoport 2012; Mak et al. 2014; Morgan, Orzen, \& Sefton 2009; Rapoport, Mak, \& Zwick 2006; Rapoport et al. 2009, 2014; Selten et al. 2007) has focused on network games with negative externalities. In contrast, ours is the first experimental study of cost-sharing in traffic network games with positive externalities.

Our study is particularly concerned with whether - and if so, how - network users might achieve collectively the efficient (socially optimal) Nash equilibrium through repeated play of a cost-sharing network game when the stage game has Pareto rankable equilibria. The socially optimal equilibrium may naturally be viewed as the optimal outcome subject to the constraint that the solution is "stable" in the sense that no agent has an incentive to unilaterally deviate from it once it is proposed (e.g., by a network designer) or, alternatively, reached by some process of adaptive learning. Our findings show that the likelihood of subjects converging towards the efficient equilibrium depends crucially on whether the payoff functions are common knowledge. In Condition Homogeneous, where travel costs were the same and commonly known among users, half of the groups converged towards the socially efficient equilibrium. In Condition Heterogeneous, where the variable travel cost of private transportation was only 
privately known, nine of the ten groups converged towards the inefficient equilibrium. We further suggest that convergence towards efficiency was facilitated by: (1) the existence of an intermediate equilibrium choice, and; (2) strategic teaching by which farsighted (sophisticated) players made route choices that significantly decreased their short-run payoff in order to signal their willingness to join public transportation and thereby achieve long-run benefits. These signals could facilitate the migration of the route choices of other group members over iterations of the stage game towards the efficient equilibrium.

We place special emphasis on the dynamics of play, in particular dynamics that converge to socially optimal outcomes (see, e.g., Balcan, Blum, \& Mansour 2013; Charikar et al. 2008). We note that when experimental games are iterated in time, players may change their behavior over iterations, exhibiting behavior that simultaneously depends on their growing understanding of the structure of game and on revisions of their beliefs about future behavior of other players in the system. Most of the previous literature attempted to explain the dynamics of play in experimental games in terms of reinforcement learning, belief learning, regret minimization, and best-response behavior (cf. Balcan 2011; Balcan et al. 2013; Camerer 2003, Chapter 6; Erev, Ert, \& Roth 2010; Nisan et al. 2007, among other examples). As noted by Balcan et al. (2013), these learning models are myopic and therefore do not fully capture the information that the players may have prior to the game or possibly acquire during the game about its overall structure, or the farsighted behavior of the users when the stage game is iterated in time. Balcan et al. mention two barriers to simple dynamics performing well in accounting for decision behavior. The first is computational; we do not know how convergence to some outcome, if reached at all, depends on the particular parameters of the experiment. The second barrier is incentive-based. Even if an efficient solution is known by the players, there is the issue of whether the players would individually be willing to play it. This may depend on the beliefs that the players acquired in previous periods about the rationality of the other players, the strategies that other players may be expected to employ in the future, and the degree that other players may be trusted to adhere to tacit agreements if, indeed, they have been reached. Moreover, to gain tractability, existing models often assume homogenous agents, whereas the experimental literature on repeated interactive behavior in large groups presents ample evidence for considerable individual differences (e.g., Gisches \& Rapoport 2012; Selten et al. 2007). Our perspective is that if a network game has multiple equilibria that are Pareto rankable, as in our study, then players have the option of sending signals, frequently at a high short-term cost to themselves, about their intention to shift group behavior from an inferior equilibrium to a more efficient equilibrium. We later refer to such behavior as strategic teaching. Some players may intrinsically be more inclined to send such signals than others. Depending on their number, these players may be critical in the convergence of the group towards an efficient outcome. We shall be reporting evidence in support of this kind of dynamics. 
The rest of the paper is organized as follows. Section 2 outlines the theoretical background of fair costsharing allocation mechanism that we employed in our experiment and the general design of our experiment. Section 3 reviews recent relevant literature on route choice. Section 4 describes the experiment. Sections 5 and 6 report the results of the experiment, including basic data analysis followed by in-depth analysis of the dynamics of play. We conclude the paper with Section 7, which includes further discussion of our findings and proposes ideas for future research.

\section{Theoretical Background and General Experimental Design}

\subsection{Fair Cost-sharing Allocation Mechanisms}

A cost-sharing allocation mechanism may be viewed as the underlying protocol of play that determines how much a network that serves multiple users will cost to each of them. Theoretical research in this area has been mostly conducted by computer scientists interested in communication networks (e.g., Anshelevich et al. 2003, 2008; Balcan et al. 2013; Harks \& Miller 2011). In the simplest protocol, each user $i, i=1,2, \ldots, k$, has a pair of nodes $\left(O_{i}, D_{i}\right)$ in a directed graph that she wishes to connect. She does so by choosing a path $S_{i}$ that originates at $O_{i}$ and terminates at $D_{i}$. The cost-sharing allocation mechanism then charges user $i$ a cost of $C_{\mathrm{i}}\left(S_{1}, S_{2}, \ldots, S_{k}\right)$, implying that the cost of user $i$ may depend on the choice of paths by the other users. In our experiment, we focus on a very natural allocation mechanism where the cost of each edge is shared equally by all the users whose paths contain it. That is:

$$
C_{i}\left(S_{1}, S_{2}, \ldots, S_{k}\right)=\sum_{e \in S_{i}} \frac{c_{e}}{\left|\left\{j: e \in S_{j}\right\}\right|},
$$

where $c_{e}$ is the cost associated with traversing edge $e$ and $|x|$ is the number of elements in set $x$. This equal ("fair") cost-sharing allocation mechanism can be rationalized by economic theorizing in the following ways: (1) it can be derived from the Shapley value, possibly the best known solution concept for cooperative games (cf. the theoretical discussion in Moulin \& Shenker 2001 and Chen \& Roughgarden 2009); and (2) it can be shown to be the unique cost-sharing scheme that satisfies a set of different axioms (see Feigenbaum, Papadimitriou, \& Shenker 2001, and Herzog, Shenker, \& Estrin 1997). The sum of the costs in the union of all paths $S_{i}$, is completely paid for by the users under this mechanism:

$$
\sum_{i=1}^{k} C_{i}\left(S_{1}, S_{2}, \ldots, S_{k}\right)=\sum_{e \in \cup_{i} S_{i}} c_{e}
$$

In our experimental setup, we take the fair cost-sharing allocation mechanism as given, and study behavioral route choices in anticipation of the fact that travelers on the same route would end up sharing costs equally. The cost-sharing stage, in the context of our experimental setup, may therefore be seen as a 
sequential game's final stage that was not actually played by the subjects, while the cooperative-solutionbased outcome of that stage determines the functional forms of the game payoffs.

Example. Following examples in Anshelevich et al. (2008) and Roughgarden and Tardos (2007), consider a directed network with $k$ players. All the players share the same destination $D$, but each player $i$ starts from her specific origin $O_{i}$. Each player may choose a private path $\left(O_{i} \rightarrow D\right)$ and incur the cost of travel 1/i. This choice and its outcome are not affected by the choices of other group members. Alternatively, player $i$ may choose the public path $\left(O_{i} \rightarrow V \rightarrow D\right)$ and incur the endogenously determined cost $(1+\mathcal{E}) / m$, where $\varepsilon>$ 0 is arbitrarily small, and $m(1 \leq m \leq k)$ is the number of players choosing this path. We assume that decisions are made in the order $1,2, \ldots, k$, and that each player is fully informed of the decisions made by the players who precede her in the sequence. It is to the benefit of all the $k$ players to choose the public path $\left(O_{i} \rightarrow V \rightarrow D\right)$, whose cost $1+\varepsilon$ is then divided equally among them. However, this solution is not a Nash equilibrium, as player $k$ can reduce her cost from $(1+\mathcal{E}) / k$ to $1 / k$ by unilaterally switching from the public to the private path $\left(O_{k} \rightarrow D\right)$. Arguing inductively for players $k-1, k-2, \ldots, 1$ shows that the unique Nash equilibrium of this cost-sharing game is the outcome in which each player chooses the private path $O_{i} \rightarrow D$. The cost of this outcome is $H(k)=1+1 / 2+1 / 3+\ldots+1 / k$, which is roughly equal to $\ln (k)$.

Models like the above example are often characterized by the ratio of the solution quality at the best Nash equilibrium relative to the quality of the solution maximizing social welfare (a number equal to or larger than 1); this ratio has been termed the price of stability $(\mathrm{PoS})$ as it captures the problem of optimization subject to the equilibrium constraint. It can be compared to the older and larger line of theoretical research in algorithmic game theory on the price of anarchy (PoA), which is the ratio of the solution quality of the worst Nash equilibrium relative to the quality of the solution maximizing social welfare. It is easy to verify that $\mathrm{PoA} \geq \mathrm{PoS} \geq 1$ (see Mak \& Rapoport 2013 for a discussion of potential applications of these concepts in social dilemma research). In the above example, the two indices of inefficiency coincide: $\mathrm{PoA}=\mathrm{PoS}=H(k) /(1+\mathcal{E})$; for example, if $k=10$, then $\mathrm{PoS}=2.93 /(1+\mathcal{\varepsilon})$.

\subsection{General Experimental Design}

The design of our experiment incorporates the fair cost-sharing allocation mechanism in an iterated transportation network game, where the choice of each user is between alternative modes of transportation. Specifically, on each round of the game, each user in our experiment had to choose independently among three alternative modes of transportation: a private route connecting a common origin and common destination $\left(O_{i} \rightarrow D\right)$, a two-edge public route $\left(O_{i} \rightarrow S H \rightarrow D\right)$ in which the cost of the edge $(S H \rightarrow D)$ had to be shared by all the users who made the same choice, and yet another two-edge public route $\left(O_{i} \rightarrow C P \rightarrow D\right)$ in which the cost of the edge $(C P \rightarrow D)$ also had to be shared by the users who 
traversed it (the symbols $O_{i}, D, S H$, and $C P$ stand for origin of player $i$, destination, shuttle, and carpool, respectively). The costs associated with traversing edges $\left(O_{i} \rightarrow S H\right)$ and $\left(O_{i} \rightarrow C P\right)$ were commonly known.

Our two experimental conditions differ from one another in the cost associated with the choice of the private mode of transportation. In Condition Homogeneous, the cost of link $\left(O_{i} \rightarrow D\right)$ was homogeneous among the users, that is, it was a constant with the same value for all the $n$ users. In Condition Heterogeneous, this cost was heterogeneous among the users; specifically, it was a real-valued number drawn randomly from a distribution $f\left(O_{i} \rightarrow D\right)$. The cost of user $i$ traversing link $\left(O_{i} \rightarrow D\right)$ was private knowledge to her, but the distribution $f\left(O_{i} \rightarrow D\right)$, was common knowledge.

\section{Relevant Literature}

\subsection{Experiments on Congestible Networks}

The paradoxical discovery of Braess (1968) that expansion of a congestible network by adding another connecting link may under certain circumstances result in degradation of performance of all users, together with the growing interest in other paradoxes of congestible transportation networks (e.g., the Down-Thompson paradox), have instigated a large body of research. Theoretical research on route choice in network games, which may or may not be susceptible to the Braess Paradox (BP), has been pursued mostly in computer science (see e.g., Cominetti et al. 2006, 2009; Fotakis, Kontogiannis, \& Spirakis 2005; Roughgarden 2005; Roughgarden \& Tardos 2007).

More directly pertinent to the present paper is the experimental investigation of route choice in congestible networks. These experiments simulate and test models of route choice in the computercontrolled environment of the laboratory, where financially motivated subjects are recruited to participate with payoff contingent on their performance. A major advantage of this approach, which we pursue in the present paper, is that the parameters of the cost-sharing games under investigation (e.g., link cost functions, information structure) may be systematically manipulated for model testing.

Previous experiments in the literature have investigated network games with finite user populations in which multiple participants independently chose single $(O \rightarrow D)$ paths. Selten et al. (2007) studied day-today route choice in a stylized network with two parallel routes. They reported aggregate route choices that are accounted for quite accurately by the equilibrium solution, and fluctuations around the mean observed choice frequencies that do not seem to diminish with experience. Helbing (2004) repeated the same experiment with more iterations of the stage network game and additional experimental conditions to better understand the reasons for the large period-to-period fluctuations in route choice. Rapoport et al. (2009) conducted an experiment on route choice that, like in the game originated by Braess, included two 
parallel routes each consisting of two connected links, one with a fixed cost and the other with a variable cost (basic network). They also constructed and experimentally studied an augmented network by adding a single cost-free link that connected the two links with the variable costs. Rapoport et al. reported that the mean route choices in the basic network converged very quickly to equilibrium. However, it took their subjects all 40 iterations to choose the new route in the augmented network that connects the two variable-cost links and, consequently, abandon the two "old" parallel routes in the basic network. In doing so, the route choices of their subjects provided strong support to the BP. Additional experiments on the Braess Paradox on directed networks with a richer topology have been reported by Gisches and Rapoport (2012), Morgan et al. (2009), and Rapoport et al. (2006, 2009).

Directed network experiments have been designed to study the undesirable effects of congestion on route choice. In these cases, individual costs associated with choosing a route increase as more players choose the same route. Pursuing a radically different approach, our study focuses on transportation networks in which the aggregate cost associated with any given route is fixed regardless of the number of users traversing this route. With users given the option of sharing the joint cost of public transportation, endogenously determined costs of individual users decrease as more of them choose to traverse the same route. Therefore, the issue of congestion and its impact on route choice is not addressed in the present study. The objective of each network user is the same as in previous studies, namely, minimization of expected cost of travel. However, this objective is best achieved by joining other users who choose traveling on the same mode of public transportation rather than choosing their own private route.

\subsection{Directed Network Experiments in Transportation Science}

Experimental studies of the effects of travel information on route choices have a longer history in transportation science. Much of the research may be traced back to the pioneering work of Mahmassani and his collaborators (e.g., Jou \& Mahmassani 1997; Srinivasan \& Mahmassani 1999). This body of research is too extensive to be reviewed here; instead, we only describe representative experiments that have been conducted in the last ten years or so. Among others, these include Avineri and Prashker (2006), Ben-Elia, Erev, and Shiftan (2008), Ben-Elia and Shiftan (2010), Ben-Elia et al. (2010), Razo and Gao (2010), and Lu, Gao, and Ben-Elia (2011). Avineri and Prashker (2006) compared reference state information, where network users updated their knowledge through reinforcement learning, to ex-ante information about expected travel time. They reported that users exposed to ex-ante information made better choices in comparison to users who only acquired experiential information. Ben-Elia et al. (2008) provided their subjects with en-route information about the variability of travel time, and reported that the informed travelers learned faster but also exhibited risk-prone behavior in the short run compared to noninformed subjects. Focusing on attitude towards risk, Ben-Elia and Shiftan (2010) reported risk-prone 
behavior in the short run but risk aversion in the long run with reinforcement learning behavior. Noting that route choice may well be sensitive to the accuracy of the information, Ben-Elia et al. (2010) conducted yet another route choice experiment in which they compared three levels of information accuracy based on the discrepancy between estimated and post choice actual travel time. Their results show that as the accuracy of the information decreased, compliance with prescriptive information decreased and choices shifted from short and riskier routes to more reliable routes.

Razo and Gao (2010) have shifted the emphasis from reinforcement learning and risk attitude to the incoming traffic information that a traveler expects to receive in the future. Their results suggest that a proper route choice model under real-time information about travel conditions should consider both myopic travelers, who exhibit no evidence for basing their route choice on future information about road conditions, and farsighted travelers who consider such information. Lu et al. (2011) compared en-route real-time information about the occurrence of traffic accidents and ex-post information about foregone payoffs in congested networks under exogenous disruptions. Their analysis suggests that en-route information reduced travel time and increased reliability under the specific setting of the experiment, and that ex-post information encouraged route switching without real-time information but suppressed it when real-time information was provided.

Jointly considered, these recent transportation experiments display considerable sensitivity to different types of travel information, access to this information, and the utility that drivers assign to travel information. The experiments are mostly descriptive in nature and, in general, not predicated on theory; variants of expected utility theory, reinforcement learning theory, and prospect theory are frequently invoked in an ad-hoc fashion to account for the experimental findings. A major drawback of these experiments is that they are individually based, ignoring congestion due to strategic interaction. Travel time in all of them is modeled by probability distributions that are assumed to be fixed over iterations of the trip and not affected by the travelers' previous choices. A possible reason for this omission is that the purpose of experimental research in transportation science might have been perceived to explain route choice in non-atomic populations, where the effect of a single traveler may, therefore, be safely ignored.

\subsection{Network Externality Studies in Economic Research}

Network externalities refer to the effect that a user of a product has on the utility of that product for other users in the same network. The classic example is the telephone; the more agents owning a telephone, the more valuable the telephone is to them. Online social networks like Twitter and Facebook also have positive externalities. Negative externalities are best illustrated by congestible networks, where delay in travel (and its consequent cost) increases as more agents choose the same route. Previous experimental 
studies on congestible networks, as reviewed earlier, are mostly about negative externalities, whereas the present study on cost-sharing focuses on positive externalities in directed networks.

The economic theory of network externalities was advanced significantly by the pioneering work of Katz and Shapiro (1985) and Farrell and Saloner (1985, 1986). Katz and Shapiro developed a static model of oligopoly to analyze markets with positive externalities, and studied two basic issues: the effect of such externalities on competition, and the incentives needed to produce compatible goods. Farrell and Saloner (1986) proposed another model, where identical firms decide sequentially whether to change to a new technology. In the context of our study, changing technology may be likened to switching to a new mode of transportation, in particular, switching from driving one's private car, where the cost of travel is constant, to some form of public transportation, where the cost of travel is shared by all the users. Farrell and Saloner showed that if all the firms would benefit from the change, then all would switch to the new technology. However, complete information of the payoff functions is typically assumed in these studies, as opposed to incomplete information scenarios as in Condition Heterogeneous of the present study. Empirical (rather than experimental) studies designed to test the effects of network externalities have been reported by Gandal (1994), Brynjolfsson and Kemerer (1996) and many others.

Experimental studies on network externalities have been rare. Examples include Devetag (2003), Mak and Zwick (2010), and Ruffle, Weiss, and Etziony (2010). These studies are typically concerned with critical mass effects and complete information about the payoff matrix among the agents. They complement our study, which involves manipulating the transparency of players' private costs to each other with a special focus on the dynamics of play. Consistent with the findings reported by Steiger and Zultan (2011), transparency in costs had significant impact on behavior in our experiment, while our analysis of dynamics sheds important light on how players might achieve collective efficiency.

\section{Description of the Experiment and Equilibrium Benchmarks}

In this section, we offer a description of the setup and implementation of the two conditions in our experiment. We also present the game-theoretic equilibria in the respective conditions, which serve as benchmarks for our data analysis.

One hundred subjects in approximately equal proportions of males and females participated in the experiment. They were primarily undergraduate students who volunteered to participate in a decision making experiment for payoff contingent on their performance. The subjects were recruited via email from a pool of students who had previously registered interest in participating in experimental studies. Fifty subjects were randomly assigned to each of the two conditions in a between-subjects design in 
which the homogeneity/heterogeneity of the players' private costs was manipulated across conditions (cf. Bachrach \& Bendoly 2011 for an overall reference on experimentation as a methodology). Throughout the experimental sessions, subjects made decisions independently through networked computers. Although they submitted their choices in a pre-specified order, since they received no information about the decisions of other subjects preceding them in the sequence the situation was effectively that of simultaneous play.

\subsection{Condition Homogeneous: Cost-sharing with Fixed Private Cost}

Design. Figure 1 displays the cost structure and interface of the computer program for Condition Homogeneous. Under a fixed-group arrangement, five groups of $n=10$ subjects played 50 identical rounds of the stage game. Each player $i$ was required to choose independently one of three modes of transportation (routes) from a fixed origin $\left(O_{\mathrm{i}}\right)$ to a fixed destination $(D)$. Because players in Condition Homogeneous were symmetric, we omit the subscript $i$ associated with the origin. The cost functions were as follow:

- Cost of the private route $(O \rightarrow D)$ was fixed at $\$ 20$ for each agent.

- $\quad$ Cost of the public route $(O \rightarrow C P \rightarrow D)$ was fixed at $\$ 10$ for segment $(O \rightarrow C P)$ and at a variable cost of $\$ 70 / m(C P \rightarrow D)$ for segment $(C \rightarrow P D)$, where $m(C \rightarrow P D)$ denotes the number of agents (out of 10) choosing this segment.

- $\quad$ Cost of the public route $(O \rightarrow S H \rightarrow D)$ was fixed at $\$ 0$ for segment $(O \rightarrow S H)$ and at a variable cost of $\$ 150 / m(S H \rightarrow D)$ for segment $(S H \rightarrow D)$, where $m(S \rightarrow H D)$ denotes the number of agents choosing this segment.

- Insert Figure 1 around here -

The instructions are very similar to those for Condition Heterogeneous (see Appendix A) except that the cost of the private route in Condition Homogeneous was kept constant at \$20. In each round, subjects chose one of the three routes by clicking on one of the options in the window "Please make Your Choice". Complete information about the distribution of choice and the individual payoff associated with the choice was provided at the end of each round. Individual payoff (rather than cost of travel) per round was computed by the function Payoff $=\$ 60 /$ cost. Subjects were paid in cash their payoffs in five randomly chosen rounds (see Appendix A) and then dismissed from the laboratory; this type of payment mechanism is quite common in the experimental literature (e.g., as can often be found in the studies cited in Camerer 2003). If subjects were paid for all the played games, then their decisions could be affected by their 
cumulative wealth effects as the session progressed (see, e.g., Cox, Sadiraj, \& Schmidt 2014), while, at the same time, the specific payoff of each round would be perceived as bearing little marginal impact to the total payment. Paying subjects for their performance in a small number of randomly chosen rounds but not so small as to make the role of chance too prominent in determining payoffs - could prevent wealth effects and instill the perception that every round "could matter".

Including a show-up fee of $\$ 5$, the mean individual payoff in Condition Homogeneous was $\$ 20.44$. The decision problem was framed in terms of costs rather than payoffs because the numerical differences between different scenarios are more pronounced if expressed as losses rather than gains.

Equilibria. The cost-sharing allocation game in Condition Homogeneous has three pure-strategy equilibria:

- A "bad" inefficient equilibrium, where all $n$ subjects choose route $(O \rightarrow D)$ at a cost of $\$ 20$, resulting in a payoff of $\$ 3$ per player.

- An "intermediate" equilibrium, where all $n$ subjects choose route $(O \rightarrow C P \rightarrow D)$ at a cost of $\$ 17$, resulting in a payoff of $\$ 3.53$ per player.

- A "good" efficient equilibrium, where all $n$ subjects choose the route $(O \rightarrow S H \rightarrow D)$ at a cost of $\$ 15$, resulting in a payoff of $\$ 4$ per player.

Consequently, $\mathrm{PoS}=1$ and $\mathrm{PoA}=4 / 3$. The major hypothesis is that with experience players converge towards one of the three equilibria. Another objective is to account for the evolution of play over iterations of the stage game.

A major difference between the two public routes is in the costs associated with out-of-equilibrium behavior that might lead to interesting dynamics as the stage game is iterated in time. Consider, for example, the case where all ten players chose the private route $(O \rightarrow D)$ and each incurred the cost of \$20. If player $i$ contemplated switching from the private to the public route $(O \rightarrow C P \rightarrow D)$, possibly with the intention of inducing most or all other nine players to do the same, then her cost of travel would increase fourfold from $\$ 20$ to $\$ 80$. If she contemplated switching to the other public route $(O \rightarrow S H \rightarrow D)$, then her cost would increase by a higher factor of 7.5 from $\$ 20$ to $\$ 150$. Similarly, if two players simultaneously switch from $(O \rightarrow D)$ to $(O \rightarrow C P \rightarrow D)$, then each would incur a cost of $\$ 45$ (rather than $\$ 20)$, whereas if both switch simultaneously to route $(O \rightarrow S H \rightarrow D)$, then each of them would incur a higher cost of $\$ 75$. Switching from the private route to either of the two public routes pays off only if at least eight out of the ten players switch to the same route. If all ten players switch from the private route to either the carpool 
or shuttle, then clearly the latter is the preferred option. Realizing that unilateral switching to the shuttle was considerably more costly than switching to the carpool, a farsighted player might switch to the carpool, and if successful in inducing other players to do so without incurring a relatively high cost for herself, wait for some other group member to incur the cost of a subsequent switch to the shuttle. This is just one of several possible dynamics that might result in the switching patterns that we propose to study below.

\subsection{Condition Heterogeneous: Cost-sharing with Randomly Determined Private Costs}

Design. The network game in Condition Heterogeneous (see Appendix A for the instructions) was identical to that in Condition Homogeneous with one major difference: the cost of choosing the private route was heterogeneous among the players; specifically, it was variable rather than fixed and privately rather than commonly known among the ten group members.

- Cost of the private route $\left(O_{i} \rightarrow D\right)$ was drawn randomly and independently for each subject from the uniform distribution on the interval [16, 24]. Note that the mean of the distribution of individual travel costs is equal to the fixed cost (\$20) for choosing the private route in Condition Homogeneous.

- Cost of the public route $\left(O_{i} \rightarrow C P \rightarrow D\right)$ was fixed at $\$ 10$ for segment $\left(O_{i} \rightarrow C P\right)$ and at a variable cost of $\$ 70 / m(C P \rightarrow D)$ for segment $(C \rightarrow P D)$.

- Cost of the public route $\left(O_{i} \rightarrow S H \rightarrow D\right)$ was fixed at $\$ 0$ for segment $\left(O_{i} \rightarrow S H\right)$ and at a variable cost of $\$ 150 / m(S H \rightarrow D)$ for segment $(S H \rightarrow D)$.

Including a show-up fee of $\$ 5$, the mean individual payment to subjects in Condition Heterogeneous was $\$ 19.15$.

Equilibria. The cost-sharing network game in Condition Heterogeneous has three pure-strategy equilibria:

- A "bad" inefficient equilibrium, where all $n$ players choose route $\left(O_{i} \rightarrow D\right)$ resulting in mean payoff per player:

$$
\frac{\$ 60}{24-16} \int_{16}^{24} d x / x=\$ 3.04
$$

- An "intermediate" equilibrium, where a player chooses route $\left(O_{i} \rightarrow D\right)$ if her private cost of choosing this route is smaller than $c^{*}=\$ 17.1$, and route $\left(O_{i} \rightarrow S H \rightarrow D\right)$, otherwise; the critical cost $c^{*}$ is the unique solution to the following equation: 


$$
\frac{60}{c^{*}}=\sum_{r=0}^{9}\left\{C_{r}^{9} p^{*^{r}}\left(1-p^{*}\right)^{9-r} \cdot \frac{60}{[150 /(r+1)]}\right\},
$$

where

$$
p^{*}=\frac{24-c^{*}}{24-16}=\frac{24-c^{*}}{8}
$$

Under this equilibrium, on average $13.8 \%[=100 \cdot(17.1-16) /(24-16)]$ of the choices would be the private route, and $86.3 \%$ of the choices would be shuttle. The expected payoff of each player, conditioned on her choosing $\left(O_{i} \rightarrow S H \rightarrow D\right)$, would be $\$ 60 / c^{*}=\$ 3.51$. The expected payoff of a player conditioned on her choosing route $\left(O_{i} \rightarrow D\right)$ would be

$$
\frac{\$ 60}{17.1-16} \int_{16}^{17.1} d x / x=\$ 3.63
$$

Further calculations show that the overall mean payoff would be $\$ 3.53$ under this equilibrium.

- A "good" efficient equilibrium, where all $n$ subjects choose the route $\left(O_{i} \rightarrow S H \rightarrow D\right)$ at a cost of $\$ 15$, resulting in an average payoff of $\$ 4$ per player.

Unlike Condition Homogeneous, unanimous choice of the carpool was no longer in equilibrium; in fact, we have found no pure-strategy equilibrium with a positive expected number of players choosing the carpool. As such, $\mathrm{PoS}=1$ and $\mathrm{PoA}=4 / 3.04=1.32$.

Each of the cost-sharing games in the two experimental conditions has three pure-strategy equilibria which are Pareto rankable. The two games differ from previous route choice experiments with multiple equilibria (typically in the millions) which are Pareto unrankable. The basic choice of each player in the present study was between private or public transportation. The cost associated with private transportation was determined solely by the player; it did not depend on the choices made by other group members. In order to incur a smaller cost associated with public transportation, players had to develop some sort of signaling system through their decisions in order to move collectively across rounds to a better equilibrium. Signals might easily be misread, misinterpreted, or simply ignored particularly if there was no way for players to associate a given signal with a specific player. Equally important is that unilateral deviations from choice of the private route carried with them costs that, depending on the number of players responding to the signal and how quickly they responded, might be substantial. Under the present design, with subject position in the sequence of choosing routes differing from one round to another, and 
cost functions associated with the choice of carpool or shuttle that are rather steep, convergence to the choice of public transportation seems highly unlikely.

\section{Basic Analysis of the Results}

We begin by reporting a series of basic data analyses for our two experimental conditions. We shall often compare equilibrium solutions with the observed patterns of choices in our data. There is no a priori assurance that route choices in any particular group would converge to equilibrium upon repeated play, or converge to any steady outcome at all. Nevertheless, as we shall report later, choices in almost every group did, in fact, converge to one of the equilibria upon repeated play.

\subsection{Overall Observations}

\section{— Insert Figure 2 around here -}

As an illustration, Figure 2 exhibits route choices of all the ten members of Group 4 in Condition Homogeneous. The rows correspond to the 50 rounds of play, and the columns to the ten group members. Group 4 was chosen randomly; Figure 2 is by no means typical as the dynamics of play varied considerably between groups. It illustrates the rate of individual switches in route choice and the subsequent challenge of choosing statistics that capture the forward looking behavior, if at all, of some subjects. For example, Subject 7 chose the shuttle on round 1 and never switched her decision for the entire 50-round session. Contrast her behavior with Subject 3, who switched her route choices, alternating between the private route, shuttle, and carpool, until finally converging on round 35 to the private route. Once nine of the ten subjects in Group 4 converged on round 31 towards the shuttle, with one exception we observe no switching from the shuttle to the carpool or private route..

\section{- Insert Figures 3 and 4 around here -}

Aggregating the data across the subjects in each group, Figures 3 and 4 portray the evolution of route choices in each group of the two experimental conditions by plotting the number of subjects choosing each of the three routes in each round. Two major observations warrant mentioning:

(1) Almost all the groups converged towards one of the three equilibria in the later part of the session. Specifically, Groups 4, 5, 7, 9, and 10 in Condition Homogeneous (Figure 3) converged towards the "good" $(O \rightarrow S H \rightarrow D)$ equilibrium, which was also the efficient outcome, whereas Groups 1, 2, 3, 6, 8 converged towards the "bad" $(O \rightarrow D)$ equilibrium, which was the least efficient outcome. With a single exception, nine of the ten groups in Condition Heterogeneous (Figure 4) converged towards the "bad" equilibrium, but the distribution of route choices typically exhibited more instability than in 
Condition Homogeneous. Group 8 in Condition Heterogeneous exhibited significant instability even towards the end of the session and did not converge distinctly to any equilibrium.

(2) The dynamics of play changed considerably from group to group. The evolution of route choices was hardly monotonic across rounds, especially with those groups in Condition Homogeneous that eventually converged towards the efficient equilibrium. We report more in-depth analysis of the dynamics in Section 6.

— Insert Table 1 around here —

\subsection{Route Choice Distributions in the Initial and Final Rounds}

In the following discussion, we examine a number specific issues related to these overall observations. Table 1 presents the distributions of choices of the private route, carpool, and shuttle in round 1 by group and condition; the cells for the groups that eventually converged towards the shuttle equilibrium are shaded in grey. Chi-square tests for every group could not reject the null hypothesis that the three modes of transportation were chosen randomly with equal probability in round 1 ( $p>0.2$ in all tests). Random choice of route in round 1 most likely reflects the novelty of the fair cost-sharing allocation mechanism, inexperience, a "wait and see" attitude, or some combination of the above.

We also conducted a logistic regression with group as the unit of analysis in which the dependent variable is 1 , if the group converged towards the shuttle equilibrium, and 0 , otherwise, while the two independent variables are the number of subjects choosing private route in round 1 and the number of subjects choosing shuttle in round 1 , respectively. The regression did not yield an estimated coefficient for any of the two independent variables that is significantly different from zero ( $p>0.4$ for both coefficients). This corroborates our finding above that the initial choice distribution was not a good predictor of equilibrium convergence.

Table 2 presents the percentages of choice in the final ten rounds of the session by group in both experimental conditions; as with Table 1, the cells for the five groups that eventually converged towards the shuttle equilibrium are shaded in grey. Consistent with Figure 3, Table 2 shows that five of the ten groups in Condition Homogeneous converged towards the inefficient private route equilibrium and five other groups to the efficient shuttle equilibrium. In contrast, consistent with Figure 4, nine of the ten groups in Condition Heterogeneous converged towards the inefficient equilibrium, with at least $80 \%$ of choices being the private route in the final ten rounds. Only Group 8 did not converge to any equilibrium with choices of its members being split evenly between the private route and carpool in the final ten rounds. 


\section{Analysis of Dynamics}

In this section, we report the results of an analysis of the dynamics of play in our two experimental conditions. We first highlight differences in system dynamics across conditions and types of equilibrium convergence in terms of evolution of route choice distributions (Section 6.1) and switching behavior (Section 6.2). Then, we focus on analyzing players' choices with respect to whether they were best responses to other players' previous choices (Section 6.3), and use our results to explain why some groups in Condition Homogeneous converged towards the shuttle equilibrium while others converged towards the private route equilibrium. Although typically not very successful, strategic teaching was used by some of the subjects in order to lead the group to convergence to the efficient equilibrium. A detailed analysis of the role of strategic teaching in the dynamics of play is presented in Section 6.4. Based on this analysis, and more speculative in nature, Section 6.5 outlines a conceptual model to capture the behavioral patterns observed in the study.

— Insert Table 3 around here -

\subsection{Evolution of Route Choice Distributions}

We divided the 50 rounds into five blocks of ten rounds each and then computed the percentage of choices by block. In the process, we aggregated the results over all groups for Condition Heterogeneous but made a distinction between groups that converged towards either the good or bad equilibria in Condition Homogeneous.

Table 3 presents the results. It displays systematic patterns of dynamic behavior. The percentage of rounds in which the bad (private route) equilibrium was chosen by Groups 4, 5, 7, 9, and 10 (set 1) declined sharply over blocks from 45.0 in block 1 to 2.2 in block 5 . In contrast, the same percentage increased from 45.8 to 96.4 in Groups 1, 2, 3, 6, and 8 (set 2) in Condition Homogeneous and from 53.6 to 89.9 over all ten groups in Condition Heterogeneous. In each case, the trend over blocks is monotonic. The percentage of rounds in which the good equilibrium (shuttle) was chosen exhibits the opposite patterns: a monotonic increase over blocks from 23.8 to 97.8 percent in set 1 , a monotonic decrease from 32.4 to 0.6 (practically zero) in set 2, and a monotonic decline from 14.8 to 0.9 in Condition Heterogeneous. Chi-square tests for the distributions in Table 3 show that, for every block, the groups that converged towards the shuttle equilibrium had significantly different choice percentages from the corresponding percentages among other groups in Condition Homogeneous as well as those in Condition Heterogeneous ( $p<0.01$ in all tests). Moreover, groups that converged towards the bad equilibrium had 
significantly different route choice distributions in every block compared with the groups in Condition Heterogeneous ( $p<0.01$ in all tests). This suggests that the dynamics were different between the two experimental conditions, and were different among groups that converged towards the two different equilibria in Condition Homogeneous.

- Insert Table 4 around here -

On average, convergence to equilibrium took place at about the same rate across different types of convergence and experimental conditions. Table 4 lists the mean round-to-round change in number of subjects choosing each route averaged within every block among the groups converging towards each type of equilibrium and in each condition, respectively. Note that round-to-round changes in these numbers were generally just one or two, and often varied between increase (positive change) and decrease (negative change), so that the entries in the table do not exceed one. The average convergence rate towards equilibrium (shuttle for five groups in Condition Homogeneous, and otherwise the private route) was around 0.13 regardless of type of group. Further $t$-tests show that these average rates do not differ significantly among any pair of the three classes of groups in Table 3 ( $p>0.4$ in all tests). However, a closer inspection of Table 4 shows that convergence took place most quickly early in the session, if the equilibrium reached was the inefficient equilibrium, while the convergence took place most quickly in the middle of the session, if the equilibrium reached was the efficient equilibrium. This is consistent with Figures 3 and 4 and Table 3, which show that groups that converged towards the efficient equilibrium did so typically after 20 rounds or so of significant instability; on the other hand, groups that converged towards the inefficient equilibrium typically had around half of their choices being the private route early in the session.

\subsection{Switching Behavior}

— Insert Figure 5 around here -

Given the initial route choice distributions, convergence could not have been achieved without subjects switching their decisions from round to round. Figure 5 displays the evolution of the percentage of switches, with a distinction between types of equilibrium convergence in Condition Homogeneous. The figure shows that, as expected from the convergence findings in Table 1, the frequency of switches in both conditions markedly decreased across rounds. It also shows that: (1) the evolution of switching frequencies did not differ by the type of equilibrium convergence in Condition Homogeneous; and (2) subjects in Condition Heterogeneous switched their decisions more frequently (almost twice as much) than in Condition Homogeneous, and remained relatively frequent switchers towards the end of the session thereby rendering the dynamics unstable. 
In Appendix B, we report further analysis that makes a distinction between switches towards more versus less efficient equilibria. The analysis shows that groups that converged towards the inefficient equilibrium tended to switch more towards less efficient equilibria (e.g., switching from carpool to private or from shuttle to private) than the other way round, while we have the opposite observation for the groups that converged towards the efficient equilibrium.

Individual switching patterns. Previous results of route choice experiments by Selten et al. (2007), Helbing (2004), Rapoport et al. (2008, 2009), and Gisches and Rapoport (2012) suggest substantive between-subject variability in the frequency of switches. In Condition Homogeneous, the individual number of switches (maximum 49) varied from 0 (Subject 8 in Group 3) to 26 (Subject 2 in Group 7), whereas in Condition Heterogeneous it varied from 2 (Subject 5 in Group 4) to 35 (Subject 4 in Group 10). In Condition Homogeneous, the mean number of switches among players in groups that converged towards the shuttle equilibrium was 5.64, while the corresponding mean was 5.76 among players in the other five groups. These two means are not significantly different from each other by $t$-test (where group is the unit of analysis to ensure independence of data points), $p>0$.9. A Kolmogorov-Smirnov test cannot reject the hypothesis that the distributions of switch frequencies are significantly different between groups that converged towards the two different equilibria, $p>0.3$. This suggests that switching frequencies alone, or individual player characteristics that might have led to differences in switching frequencies, could not explain the different types of equilibrium convergence in Condition Homogeneous.

Meanwhile, the mean number of switches among players in Condition Heterogeneous was 10.7, which is significantly larger than the corresponding mean of 5.7 in Condition Homogeneous by $t$-test with group as the unit of analysis $(t(18)=3.85, p<0.01)$. This difference aggregated upwards to create the difference in overall switching frequencies observed in Figure 5. We conclude that uncertainty about cost of the private route resulted in subjects in Condition Heterogeneous switching their routes more frequently than in Condition Homogeneous. Consistent with Figure 5, further statistical examination confirms that individual switching frequencies tended to decrease across the session; this conclusion applies to both switches towards efficient and inefficient equilibria.

\subsection{Best Response Analysis}

The analysis reported in the previous two sections focused on route choice distributions and frequency of switches. An in-depth look at the dynamics requires an investigation into how players' choices might have been affected by the choices of other players. An approach along this direction is to investigate whether the decisions in the experiment best responded to other players' decisions in the previous round.

— Insert Tables 5 and 6 around here - 
To begin with, we recorded for every player her ex post best response in every round, given the realized decisions of all other players. ${ }^{1}$ Table 5 lists the percentages of these best responses by block, condition, and type of equilibrium convergence. We also rank order the three routes according to the efficiency of the associated equilibrium, such that the shuttle is ranked higher than the carpool, which, in turn, is ranked higher than the private route. We then examine, for every round $t>1$, if the player's choice in round $t$ was the same as her ex post best response in round $t-1$, or if not, whether the choice was ranked higher or lower than the ex post best response. ${ }^{2}$ The results of the analysis are reported in Table 6 , which lists the percentage frequencies of route choices relative to the ex post best response for the previous round (note that block 1 in Table 6 begins from round 2 rather than round 1). Further analysis leads to the following findings, which we summarize under three categories of route choices:

(1) Choosing a lower-ranked route than the ex post best response to the previous round. These choices seem to happen rarely according to Table 6 . However, a low proportion could be partly due to there being many observations in which the private route was the ex post best response - there were no lower-ranked route to choose in those observations, but they still contributed towards the base count. This was especially the case among groups that converged towards the inefficient equilibrium. If we make an adjustment by limiting the base counts to only observations where the ex post best response was not the private route, subjects chose a lower-ranked route choice in $4.13 \%$ (out of 1696 observations), $19.7 \%$ (out of 76 observations), and 22.8\% (out of 136 observations) of the times across all blocks among the three types of groups from left to right in Table 6, respectively. The adjusted proportion is especially low with groups that converged towards the efficient equilibrium. Pairwise $t$-tests, with group as the unit of analysis, confirm that the proportion is significantly lower among groups that converged towards the efficient equilibrium than among the groups in Condition Heterogeneous, at $p<0.01 .^{3}$

(2) Choosing the ex post best response to the previous round. Table 6 suggests that subjects most often best responded to the previous round, especially from block 2 onwards. Further $t$-tests with group as the unit of analysis shows that, across all blocks, the proportion of times when subjects best responded to the previous round is higher than $65 \%$ at $p<0.05$ in every one of the three types of

\footnotetext{
${ }^{1}$ There were 51 observations in Condition Homogeneous and two observations in Condition Heterogeneous, out of a total of 2000, where a player would have two equally preferable ex post best responses in a round. In those cases, the tie was always between private route and carpool. We always broke the tie in favor of private route; breaking the tie otherwise has no impact on our major conclusions.

${ }^{2}$ When defining round $t$ best response to round $t-1$ (which then includes strategic teaching behavior; see later) in Condition Heterogeneous, we make the following adjustment: a player's round $t$ best response to round $t-1$ would be her optimal choice given her private route cost in round $t$, and the choices of other players in round $t-1$.

${ }^{3} \mathrm{~A}$ similar $t$-test involving the groups in Condition Homogeneous that converged towards the inefficient equilibrium cannot be conducted because in only one group among them the ex post response was not the private route.
} 
groups in Table 6. Moreover, pairwise $t$-tests show that this proportion is not significantly different among the three types of groups ( $p>0.1$ in all relevant tests).

(3) Choosing a higher-ranked route than the ex post best response to the previous round. According to Table 6 , these happen around $13 \%$ to $17 \%$ of the times across all blocks among each of the three types of groups. Even among groups that converged towards the efficient equilibrium - where the ex post best responses in later rounds were mostly shuttle, so that subjects did not have any higherranked route choice to choose - the proportion remains similarly high as with the other groups due to frequent choice of this kind in the early rounds. If we limit the base counts to only observations where the ex post best response was not the shuttle, subjects chose a higher-ranked route choice in $38.0 \%$ (out of 989 observations), $13.7 \%$ (out of 2374 observations), and 17.0\% (out of 4899 observations) of the times across all blocks among the three types of groups from left to right in Table 6, respectively. The adjusted proportion is especially high with groups that converged towards the efficient equilibrium; pairwise $t$-tests, with group as the unit of analysis, confirm that this proportion is significantly higher among groups that converged towards the efficient equilibrium than among either of the two other types of groups, at $p<0.01$.

Overall, the analysis in the present section suggests that groups that converged towards the efficient equilibrium differed from other groups by: (1) a lower tendency to choose routes that were lower-ranked than the ex post best response to the previous round; and (2) a higher tendency to choose routes that were higher-ranked than the ex post best response to the previous round. The latter tendency is especially interesting, as the relevant route choices helped migrating the groups to a more efficient equilibrium. We say that those decisions exhibited "strategic teaching," in connection with related findings from previous literature for which we offer further discussion in the next section.

\subsection{Strategic Teaching: Short-term vs. Long-term Payoffs}

In this section, we focus on the crucial question of why some groups in Condition Homogeneous converged towards the efficient equilibrium while others converged towards the inefficient equilibrium. We suggest that the answer lies in two main facilitating factors:

(1) The use of carpool as a signal to induce other players to eventually choose the efficient equilibrium choice. As shown in Table 3, the groups in Condition Homogeneous that converged towards the inefficient equilibrium had more players switching from the shuttle to private route as the session proceeded, with low interest in the carpool as an "intermediate" choice. But for groups that converged towards the efficient equilibrium, the choice of carpool was important in facilitating convergence to efficiency in the first three blocks of the session. The choice of carpool could be perceived as a safer 
substitute for shuttle for players who tried to induce (or "teach") other players to depart from choosing the private route. This intuition is supported by our best response analysis: Table 5 shows that carpool was often an ex post best response in the first three blocks for groups that eventually converged towards the shuttle equilibrium. Pairwise $t$-tests with group as the unit of analysis confirm that, over the first three blocks, there were significantly more observations where carpool was the ex post best response among groups that converged towards the efficient equilibrium, compared with either of the other two types of groups, at $p<0.05$. This is corroborated by an examination of Figure 3, which shows that in Groups 5, 7, and 9 in Condition Homogeneous - which converged towards the efficient equilibrium - carpool was the predominant choice in early rounds. In fact, the players in Groups 5 and 7 even temporarily reached the "intermediate" carpool equilibrium in some rounds. By stark contrast, carpool was hardly an ex post best response with all other groups.

(2) Strategic teaching. Although carpool and shuttle were often ex post best responses in the initial rounds among groups that converged towards the efficient equilibrium, the private route was the predominant ex post best response in those rounds (Table 5). A member of those groups would consider carpool as no less preferable ex post than the private route only if at least six other subjects chose the carpool; the corresponding number would be eight for the shuttle. But Table 1 shows that in most groups that converged towards the efficient equilibrium neither of these thresholds was reached in round 1. Thus, for convergence to the efficient equilibrium to happen subsequently, it was important for some subjects to choose carpool or even shuttle when those were not ex post best responses to the previous round. This leads to our analysis of strategic teaching, by which a player makes a more efficient equilibrium choice than the best response to other players' choices in previous rounds. In doing so, she attempts transmitting signals to other players about her willingness to coordinate to achieve efficiency in the long run, even if her choice may lead to suboptimal payoffs in the short run. Strategic teaching could thus be seen as a player's rationally motivated short-term investment for long-run payoffs that would be higher than if the same player continued to follow an inefficient equilibrium.

Strategic teaching was introduced by Fudenberg and Levine (1998) in their seminal book on learning in games. Ellison (1997) provided a theoretical treatment regarding how a single rational player could manipulate the choices of myopic players towards an efficient equilibrium in a coordination game. Camerer, Ho, and Chong (2002) noted that sophisticated players, who are matched with the same players repeatedly as in our two experimental conditions, might have an incentive to "teach" other players "by choosing strategies with poor short-term payoffs which will change what adaptive players do in a way that benefits the sophisticated player in the long run" (Camerer et al. 2002, p.139). 
Brandts and Cooper (2006) and Brandts, Cooper, and Fatas (2007) provided experimental evidence that endogenously emerging "leaders" among the players, fulfilling the function of a strategic teacher, could pull other players out of an inefficient equilibrium towards an efficient one in a coordination game with multiple Pareto rankable equilibria. Further experimental results on strategic teaching in similar games, with more investigation into how the form of the payoff matrix could affect strategic teaching, as well as successful versus unsuccessful teaching, could be found in Hyndman, Terracol, and Vaksmann (2009) and Hyndman et al. (2012), among others. Of particular interest is the finding reported by Hyndman et al. that strategic teaching is much more difficult when players have limited information about each other's payoffs. This is consistent with our findings that Condition Heterogeneous yielded less efficient outcomes than Condition Homogeneous. Note also that our experimental design offers additional evidence about strategic teaching in the case where reputation building is not possible, because individual players in our setup could not be identified.

To assess the effectiveness of strategic teaching, we say that strategic teaching behavior of degree $r>$ 0 occurred in round $t>r$ with player $i$, if and only if, in round $t-k$ for every $k=0,1,2 \ldots r-1, i$ chose a route that was ranked higher than the ex post best response route to round $t-k-1$ (with the ranks defined as in the previous section). For a simple example, consider $r=1$. According to our definition, strategic teaching behavior of degree 1 occurred in round $t>1$ with player $i$ if and only if $i$ chose carpool or shuttle in round $t$ while the ex post best response to $t-1$ was the private route, or if $i$ chose shuttle in round $t$ while the ex post best response to $t-1$ was carpool. ${ }^{4}$ Lastly, to abuse notation, define as strategic teaching behavior of degree 0 an observation in which a player's ex post best response to the previous round was private route or carpool, but the player's choice was ranked the same or lower than the ex post best response. That is, strategic teaching of degree 0 occurred when a player could have exhibited strategic teaching behavior but did not do so.

Note that a player might exhibit strategic teaching of a positive degree in round $t$ if she believed that a sufficient number of other players would have switched decisions to make a higher ranked choice optimal in round $t$. However, regardless of the player's motivation or belief, the effect of her decision on other players would have made a similar impact, all else being equal. Thus, we shall not examine such a distinction in our analysis.

— Insert Table 7 around here -

\footnotetext{
${ }^{4}$ Other definitions are possible, such as restricting strategic teaching behavior to be choosing the shuttle when the ex post best response to the previous round was otherwise; or restricting that the ex post best response to the previous round had to be the private route. The qualitative insights from the analysis remain similar with these alternative definitions.
} 
Table 7 presents the percentages of strategic teaching decisions of different degrees by condition and type of equilibrium convergence. First, observe that the number of observations with which strategic teaching was definable is smaller among the five groups that converged towards the efficient equilibrium than the other five groups in Condition Homogeneous (989 vs. 2374). This is because the ex post best responses to previous rounds were much more often the shuttle among the former groups, when strategic teaching would be undefinable. But whenever strategic teaching could be exhibited, it was not uncommon; in each of the three types of groups in Table 7 there were nine or more instances of strategic teaching of at least 15 degrees. Nevertheless, when comparing among types of groups, it is evident that strategic teaching of a positive degree occurred considerably more frequently among groups that converged towards the efficient equilibrium than either of the other two types of groups (38\% vs. $13.7 \%$ and $17 \%$ for the other two types of groups). Pairwise $t$-tests with group as the unit of analysis confirm this statistically ( $p<0.01$ in both relevant tests). Hence, whenever a player could exhibit strategic teaching, a player within the groups that eventually converged towards the efficient equilibrium was significantly more likely to do so in comparison with other groups.

Further analyses lead to the following findings:

(a) Strategic teaching was typically not successful. To illustrate this point, define a player's strategic teaching in a round as successful whenever the ex post best response of that player in the next round turned out to be ranked higher than that in the current round. Given this definition, we find that, conditioned on strategic teaching behavior having occurred, it would be successful $19.7 \%$ of the times in Condition Homogeneous among groups that converged towards the efficient equilibrium; this percentage decreased sharply to only $2.2 \%$ with the other groups in Condition Homogeneous and $4.6 \%$ among all groups in Condition Heterogeneous. Pairwise $t$-tests with group as the unit of analysis confirm that this success rate variable was significantly higher among the groups that converged towards the efficient equilibrium than either of the other two types of group ( $p<0.05$ in both tests). To further highlight these differences, among the five groups that converged towards the efficient equilibrium in Condition Homogeneous, the success rate of strategic teaching in a group was at least $6.4 \%$ and could be as high as $61.8 \%$. On the other hand, in four of the groups that converged towards the inefficient equilibrium in Condition Homogeneous, and in three of the groups in Condition Heterogeneous, no instance of strategic teaching was successful by our definition.

(b) Strategic teaching behavior adversely affected a player's payoff. We find that the number of times a player exhibited strategic teaching was significantly and positively correlated with her 
mean per round transportation cost, with correlation coefficients of $0.77,0.89$, and 0.85 , respectively ( $p<0.01$ in all three correlation analysis) for the three types of groups in Table 7 from left to right.

(c) Analysis of strategic teaching by blocks of ten rounds shows that groups that converged towards the efficient equilibrium exhibited strategic teaching significantly more often than either of the other two types of groups in block 3, but not in blocks 1 and 2 (using $t$-tests with group as the unit of analysis and significance criterion $p<0.05$ ). It appears that groups converged towards efficiency because of persistent strategic teaching behavior during the first 30 rounds or so, after which all players' choices were successfully migrated towards the shuttle.

\subsection{A Conceptual Model of Route Choice in the Experiment}

Our analysis of the dynamics of play offers a wealth of information about behavioral patterns in the experiment. In general, as the session progressed, route choice distribution stabilized with a decrease in switching frequencies. Meanwhile, convergence was relatively more "noisy" or unstable in Condition Heterogeneous than in Condition Homogeneous, resulting in switching occurring about twice more frequently overall in the former condition.

Subjects seemed to choose routes randomly in round 1 (Table 1), and then often made choices as if they were best responding to other players' choices in the previous round (Table 6). In addition, in Condition Heterogeneous they were more likely to use the private route the lower their cost of traversing that route.

A minor proportion of choices were not best responses to the previous round, but were instead the routes of more efficient equilibria. In relation to previous related literature, we call such behavior strategic teaching, as it could lead the group towards the most efficient equilibrium; in particular, the carpool was often used as a transitional choice in strategic teaching. Strategic teaching was in general not successful, but nevertheless in Condition Homogeneous it helped half of the groups to finally converge towards the efficient equilibrium. The ten groups in Condition Heterogeneous had not benefitted from the strategic teaching behavior among them to the same extent as in Condition Homogeneous; this result is consistent with previous findings that strategic teaching was much more difficult when players had limited information about each other's payoffs.

Summarizing our findings, we outline a conceptual model of route choice in the cost-sharing experiment, whereby a player's route choice in round $t(t>1)$ is primarily influenced by two classes of factors:

(1) The "hypothetical cost" of each route in round thad the player chosen that route while all other players made the same choices as they had in round $t-1$. This is, in fact, the cost that determines 
the player's ex post best response to the previous round. We propose that the lower the hypothetical cost of a route, the more attractive the route is to the player. This influence is consistent with our observations that subjects tended to best respond to the previous round.

(2) The "strategic teaching" utilities of routes that are higher-ranked than the best response to the previous round, over and above their hypothetical costs. We propose that, the higher the strategic teaching utility of a route, the more attractive the route is to the player. The utility could be affected by the player's beliefs in the future payoffs that a choice of the associated route can yield through influencing others to migrate towards the efficient equilibrium. Thus, we conjecture that the strategic teaching utilities could be functions of $t$ (since future payoffs generally decrease with $t$ ) and some summary statistics of the history of route choice distributions in the experiment, both of which might impact on the player's belief. We also conjecture that those utilities are far more sensitive to individual differences than the impact of hypothetical costs; as a result, subjects in our experiment seemed to often best respond to the previous round in general (Section 6.3), but varied sufficiently in their tendency to exhibit strategic teaching that the groups in Condition Homogeneous differed dramatically in equilibrium convergence.

A third class of factors may be considered, which denote the utilities of routes that are lower-ranked than the best response to the previous round, over and above their hypothetical costs. These factors can be seen as proxies for players' tendency to choose "safer" routes than the best response to the previous round. They would have a major impact on observed data only among groups for which the best response to previous round was often the carpool or shuttle, i.e., groups that converged towards the efficient equilibrium. But, as discussed earlier in Section 6.3, those groups would be expected to have particularly low values for these utilities. Thus, for the sake of parsimony, a third class of factors may not be necessary for a descriptive model of subjects' decision making in our context.

Previous experimental studies of congestible network games have employed stochastic learning models that involve hypothetical costs and a stationary presence of noises in decision making (e.g. Mak et al. 2014). However, the noises in decision making often decreased rapidly at the end of the session in Condition Homogeneous, suggesting that previous models may need to be adjusted for the present context with positive externalities. Modelling the strategic teaching utilities poses another level of challenge, as an in-depth understanding probably needs data about subjects' belief regarding the future impact of route choices. Further research is required to clarify these issues.

\section{Conclusions}


Our study makes a threefold contribution to the understanding of dynamic systems in which groups of agents repeatedly interact through their decisions. To our best knowledge, this is the first experimental study on directed transportation networks with positive externalities induced by cost-sharing. As our results clearly demonstrate, it is important to examine how human subjects interact with one another in such scenarios, and how the interactions might evolve with time. On the other hand, previous experimental research on directed networks focused on negative externalities induced by congestion. Our research complements that stream of studies and initiates new directions with potential applications that cut across management science, transportation science, and economics.

Secondly, our findings shed light on the factors that drive convergence to social efficiency in systems with positive network externalities in which reputation building is not possible. Specifically, we highlight the crucial role of an "intermediate" equilibrium choice in the form of carpool, as well as strategic teaching behavior by which a player makes a more efficient equilibrium choice than her best response to other players in the previous round. These results are in full agreement with those reported by Murphy et al (2006), where the binary choice that each group member has to make in real time is whether to let her group payoff increasing over time or be the first to defect and thereby rip a considerably higher payoff.

Thirdly, the fact that nine of the ten groups in Condition Heterogeneous converged towards the inefficient equilibrium, and that the dynamics were more unstable compared with Condition Homogeneous, points to the behaviorally adverse effect of non-transparent cost functions among players (see Steiger \& Zultan 2013 on comparison of different degrees of transparency). Note that the "poor" performance in Condition Heterogeneous could not be due to heterogeneous costs per se; as Ruffle et al. (2010) find out, heterogeneous payoff functions might actually lead to higher efficiency if they were transparent common knowledge. It appears to be incomplete cost information among players that contributed towards inefficiency and instability in Condition Heterogeneous. This conclusion is consistent with Hyndman et al. (2012)'s findings that strategic teaching was more difficult when players had limited information about each other's payoffs.

Future Directions. A limitation of the current study is the lack of direct evidence on the cognitive processes of subjects, especially regarding strategic teaching. Hence, a potential direction of future research is to elicit beliefs of subjects after each round and then analyze them in conjunction with their choices (see, e.g., Hyndman et al. 2009, 2012); nevertheless, a note of caution is that belief elicitation may itself have an effect on subjects' behavior in the experimental game (Ruström \& Wilcox 2009). A further possibility is to carry out in-depth surveys or interviews with subjects at the end of the session to obtain more process data. A second major direction is to investigate behavior in the context of other 
modes of cost-sharing in transportation networks. One possibility is to render the protocol of play sequential (rather than simultaneous) by letting players choose routes one after another within the same game, with some degree of observability over predecessors' choices. Thirdly, experimental investigations might reach out to networks with richer architecture where link costs exhibit a combination of congestioninduced negative externalities and cost-sharing-induced positive externalities. It would be fruitful to examine how these two types of common externalities might interact with each other in their impact on efficiency. Finally, success or failure by strategic teachers depends critically on group size, possibility of reputation building, and cost structure. This is also evident in research on the volunteer's dilemma (Diekmann 1985; Otsubo \& Rapoport 2008), where only a single player ("volunteer") is required to drastically change the group outcome. Additional research that systematically manipulates one or more of these parameters is warranted. 


\section{References}

Anshelevich, E. et al. 2003. Near-optimal network design with selfish agents. STOC'03 511-520.

Anshelevich, E. et al. 2008. The price of stability for network design with fair cost allocation. SIAM Journal of Computing 38 1602-1623.

Avineri, E., J. N. Prashker. 2006. The impact of travel time information on travelers' learning under uncertainty. Transportation 33 393-408.

Bachrach, D. G., E. Bendoly. 2011. Rigor in behavioral experiments: A basic primer for supply chain management researchers. Journal of Supply Chain Management 47 5-8.

Balcan, M.- F. 2011. Leading dynamics to good behavior. ACM SIGecom Exchanges 10 19-22.

Balcan, M.-F., A. Blum, Y. Mansour. 2013. Circumventing the price of anarchy: Leading dynamics to good behavior. SIAM Journal of Computation 42 230-264.

Ben-Elia, E., R. Di Pace, G. Bifulco, Y. Shiftan. 2010. Modelling the impacts of ATIS accuracy on travellers' route-choice behaviour and risk perception. European Transport Conference 1-19.

Ben-Elia, E., I. Erev, Y. Shiftan. 2008. The combined effect of information and experience on drivers' route-choice behavior. Transportation 35 165-177.

Ben-Elia, E., Y. Shiftan. 2010. Which road I take? A learning-based model of route choice with real-time information. Transportation Research Part A 44 249-264.

Braess, D. 1968. Über ein Paradoxon der Verkehrsplanung. Unternehmensforschung 12 258-68.

Brandts, J., D. J. Cooper. 2006. A change would do you good ... An experimental study on how to overcome coordination failure in organizations. American Economic Review 96 669-693.

Brandts, J., D. J. Cooper, E. Fatas. 2007. Leadership and overcoming coordination failure with asymmetric costs. Experimental Economics 10 269-284.

Brynjolfsson, E, C., Kremer. 1996. Network externalities in microcomputer software: An econometric analysis of the spreadsheet market. Management Science 42 1627-1647.

Camerer, C. F. 2003. Behavioral Game Theory. Princeton, NJ: Princeton University Press. 
Camerer, C. F., T.-H. Ho, J.-K. Chong. Sophisticated experience-weighted attraction learning and strategic teaching in repeated games. Journal of Economic Theory 104 137-188.

Charikar, M. et al. 2008. Online multicast with egalitarian cost sharing. SPAA 70-76.

Chen, H,-L., T. Roughgarden. 2009. Network design with weighted players. Theory of Computer Systems 45 302-324.

Cominetti, R., J. R. Correa, N. E. Stier-Moses. 2006. Network games with atomic players. In M. Bugliesi et al. (Eds.), Automata, Language and Programming. Proceedings of the $33^{\text {rd }}$ International Colloquium (ICALP), Vol. 4051. Lecture Notes in Computer Science, Part 1. Heidelberg: Springer, 525-536.

Cominetti, R., J. R. Correa, N. E. Stier-Moses. 2009. The impact of oligopolistic competition in networks. Operations Research 57 1421-1437.

Correa, J. R., N. E. Stier-Moses. 2011. Wardrop equilibria. In J. J. Cochran (Ed.), Wiley Encyclopedia in Operations Research and Management Science, New York: Wiley.

Cox, J. C., V. Sadiraj, U. Schmidt (2014). Alternative payoff mechanisms for choice under risk. Kiel Institute for the World Economy, Working Paper No. 1932.

Daniel, T. E., E. J. Gisches, A. Rapoport. 2009. Departure time in Y-shaped traffic networks with multiple bottlenecks. American Economic Review 99 2149-76.

Devetag, G. 2003. Coordination and information in critical mass games: An experimental study. Experimental Economics 6 53-73.

Diekmann, A. 1985. Volunteer's dilemma. Journal of Conflict Resolution 42 600-618.

Ellison, G. 1997. Learning from personal experience: One rational guy and the justification of myopia. Games and Economic Behavior 19 180-210.

Erev, I., E. Ert, A. E. Roth. 2010. A choice prediction competition for market entry games: An introduction. Games 1 117-136.

Farrell, J., G. Saloner. 1985. Standardization, compatibility, and innovation. The RAND Journal of Economics 16 70-83. 
Farrell, J., G. Saloner. 1986. Installed base and compatibility: Innovation product preannouncement, and predation. American Economic Review 76 940-955.

Feigenbaum, J., C. Papadimitriou, S. Shenker. 2001. Sharing the cost of multicast transmissions. Journal of Computer and System Sciences $\mathbf{6 3} 21-41$.

Fotakis, D., S. Kontogiannis, P. Spirakis. 2005. Theoretical Computation Science 348 226-239.

Fudenberg, D, D. Levine (1998). The Theory of Learning in Games. Cambridge, MA: MIT Press.

Gandal, F. 1994. Hedonic price indexes for spreadsheets and an empirical test for network externalities. The RAND Journal of Economics 25 160-170.

Gisches E., A. Rapoport. 2012. Degrading network capacity may improve performance: Private vs. public monitoring in the Braess Paradox. Theory and Decision 73 267-293.

Harks, T., K. Miller. 2011. The worst-case efficiency of cost sharing methods in resource allocation games. Operations Research 59 1491-1503.

Helbing, D. 2004. Dynamic decision behavior and optimal guidance through information services: Models and experiments. In M. Schreckenberg and R. Selten (Eds.), Human Behavior and Traffic Networks. Berlin: Springer-Verlag, 47-95.

Herzog, S., S. Shenker, D. Estrin. 1997. Sharing the "cost" of multicast trees: An axiomatic analysis. IEEE/ACM Transactions on Networking 5 847-860.

Hyndman, K., E. Y. Ozbay, A. Schotter, W. Z. Ehrblatt. 2012. Convergence: An experimental study of teaching and learning in repeated games. Journal of the European Economic Association 10 573-604.

Hyndman, K., A. Terracol, J. Vaksmann. 2009. Learning and sophistication in coordination games. Experimental Economics 12 450-472.

Jou, R.-C. H., S. Mahmassani. 1997. Comparative analysis of day-to-day trip-chaining behavior of urban commuters in two cities. Transportation Research Record 1697 163-170.

Katz, M. L., C. Shapiro. 1985. Network externalities, competition, and compatibility. American Economic Review 75 424-440.

Lu, X., S. Gao, E. Ben-Elia. 2011. Information impacts on route choice and learning behavior in a congested network. Transportation Research Record 2243 89-98. 
Mak, V., A. Rapoport. 2013. The price of anarchy in social dilemmas: Traditional research paradigms and network applications. Organizational Behavior and Human Decision Making 120 142-153.

Mak, V., E. J. Gisches, A. Rapoport. 2014. Route vs. segment: An experiment on real-time travel information in congestible networks. Forthcoming in Production and Operations Management.

Mak, V., R. Zwick. 2010. Investment decisions and coordination problems in a market with network externalities: An experimental study. Journal of Economic Behavior and Organization 76 759-773.

Monderer, D., L. Shapley. 1996. Potential games. Games and Economic Behavior 14 124-143.

Morgan, J. H., H. Orzen, M. Sefton. 2009. Network architecture and traffic flows: Experiments on the Pigou-Knight-Downs and Braess Paradoxes. Games and Economic Behavior 66 348-372.

Moulin, H., S. Shenker. 2001. Strategyproof sharing of submodular costs: Budget balance versus efficiency. Economic Theory 18 511-533.

Murphy, R. O., A. Rapoport, J. E. Parco. 2006. Breakdown in cooperation in iterated real-time trust dilemmas. Experimental Economics 9 147-166.

Nisan, N. et al. 2007. Algorithmic Game Theory. Cambridge, MA: Cambridge University Press.

Otsubo, H., A. Rapoport. 2008. Dynamic volunteer's dilemmas over finite horizon: An experimental study. Journal of Conflict Resolution 52 961-984.

Rapoport, A., E. J. Gisches, V. Mak. 2014. Distributed decisions in networks: Laboratory study of routing splittable flow. Production and Operations Management 23 314-331.

Rapoport, A., T. Kugler, S. Dugar, E. J. Gisches. 2009. Choice of routes in congested traffic networks: Experimental tests of the Braess Paradox. Games and Economic Behavior 65 538-71.

Rapoport, A., V. Mak, R. Zwick. 2006. Navigating congested networks with variable demands: Experimental evidence. Journal of Economic Psychology 27 648-66.

Razo, M., S. Gao. 2010. A rank-dependent expected utility model for strategic route choice with stated preference data. Department of Civil and Environmental Engineering, University of Massachusetts, Working Paper.

Roughgarden, T. 2005. Selfish Routing and the Price of Anarchy. Cambridge, MA: MIT Press. 
Roughgarden, T., É. Tardos. 2007. Introduction to the inefficiency of equilibria. In N. Nisan et al. (Eds), Algorithmic Game Theory. Cambridge, MA: Cambridge University Press.

Ruffle, B. J., A. Weiss, A. Etziony. 2010. Coordination and critical mass in a network market: an experimental investigation. Bar-Ilan University, Department of Economics Research Paper 2010-03.

Rustrom, E. E., N. T. Wilcox. 2009. Stated beliefs versus inferred beliefs: A methodological inquiry and experimental test. Games and Economic Behavior 67, 616-632.

Selten, R., T. Chmura, T. Pitz, S. Kube, M. Schreckenberg. 2007. Commuters' route choice behavior. Games and Economic Behavior 58 394-406.

Srinivasan, K. K., H. Mahmassani. 1999. Role of congestion and information in tripmakers' dynamic decision processes: An experimental investigation. Transportation Research Record 1676 43-52.

Steiger, E.-M., R. Zultan. 2011. See no evil: Information chain and reciprocity in teams. Jena Economic Research Papers, No. 2011-040. Jena, Germany. 
Table 1. Number of subjects choosing each route in round 1 by group and condition.*

\begin{tabular}{cccccccc}
\hline & \multicolumn{3}{c}{ Condition Homogeneous } & & \multicolumn{3}{c}{ Condition Heterogeneous } \\
\cline { 2 - 4 } \cline { 7 - 8 } Group & Private & Carpool & Shuttle & & Private & Carpool & Shuttle \\
\hline 1 & 3 & 3 & 4 & & 5 & 2 & 3 \\
2 & 4 & 5 & 1 & & 3 & 5 & 2 \\
3 & 4 & 4 & 2 & & 2 & 6 & 2 \\
4 & 0 & 4 & 6 & & 4 & 4 & 2 \\
5 & 3 & 4 & 3 & & 2 & 5 & 3 \\
6 & 6 & 0 & 4 & & 3 & 3 & 4 \\
7 & 4 & 3 & 3 & & 2 & 3 & 5 \\
8 & 3 & 3 & 4 & & 5 & 1 & 4 \\
9 & 5 & 4 & 1 & & 3 & 7 & 0 \\
10 & 2 & 4 & 4 & & 4 & 4 & 2 \\
\hline Total & $\mathbf{3 4}$ & $\mathbf{3 4}$ & $\mathbf{3 2}$ & & $\mathbf{3 3}$ & $\mathbf{4 0}$ & $\mathbf{2 7}$
\end{tabular}

* Shaded cells indicate the five groups that eventually converged towards the shuttle equilibrium.

Table 2. Choice percentages in rounds $41-50$ by group and condition.*

\begin{tabular}{ccccccccc}
\hline & \multicolumn{3}{c}{ Condition Homogeneous } & & \multicolumn{3}{c}{ Condition Heterogeneous } \\
\cline { 2 - 4 } \cline { 6 - 8 } Group & Private & Carpool & Shuttle & & Private & Carpool & Shuttle \\
\hline 1 & 96 & 3 & 1 & & 95 & 0 & 5 \\
2 & 99 & 0 & 1 & & 98 & 1 & 1 \\
3 & 100 & 0 & 0 & & 99 & 1 & 0 \\
4 & 10 & 0 & 90 & & 85 & 14 & 1 \\
5 & 0 & 0 & 100 & & 91 & 9 & 0 \\
6 & 98 & 2 & 0 & & 99 & 1 & 0 \\
7 & 1 & 0 & 99 & & 99 & 0 & 1 \\
8 & 89 & 10 & 1 & & 54 & 45 & 1 \\
9 & 0 & 0 & 100 & & 94 & 6 & 0 \\
10 & 0 & 0 & 100 & & 80 & 19 & 1 \\
\hline
\end{tabular}

*Shaded cells indicate the five groups that converged towards the shuttle equilibrium. 
Table 3. Choice percentages by block and condition.*

\begin{tabular}{cccccccccc}
\hline & \multicolumn{3}{c}{$\begin{array}{c}\text { Condition Homogeneous } \\
\text { (Group 4, 5, 7, 9, 10) }\end{array}$} & \multicolumn{2}{c}{$\begin{array}{c}\text { Condition Homogeneous } \\
\text { (Group 1, 2, 3, 6, 8) }\end{array}$} & \multicolumn{2}{c}{$\begin{array}{c}\text { Condition Heterogeneous } \\
\text { (All ten groups) }\end{array}$} \\
\hline Round & Private & Carpool & Shuttle & Private & Carpool & Shuttle & Private & Carpool & Shuttle \\
\hline $1-10$ & 45.0 & 31.2 & 23.8 & 48.8 & 18.8 & 32.4 & 53.6 & 31.6 & 14.8 \\
$11-20$ & 32.8 & 24.0 & 43.2 & 81.4 & 7.2 & 11.4 & 78.7 & 16.2 & 5.1 \\
$21-30$ & 12.0 & 16.8 & 71.2 & 93.2 & 2.8 & 4.0 & 88.0 & 9.0 & 3.0 \\
$31-40$ & 2.8 & 1.0 & 96.2 & 95.8 & 3.0 & 1.2 & 90.7 & 7.5 & 1.8 \\
$41-50$ & 2.2 & 0.0 & 97.8 & 96.4 & 3.0 & 0.6 & 89.9 & 9.2 & 0.9 \\
\hline Overall & 19.0 & 14.6 & 66.4 & 83.1 & 7.0 & 9.9 & 80.2 & 14.7 & 5.1 \\
\hline
\end{tabular}

* The entries for Condition Homogeneous were listed separately for the five groups that converged towards the shuttle equilibrium (shaded cells) and the five groups that converged towards the private route equilibrium.

Table 4. Mean round-to-round change in number of subjects choosing each route by block and condition,* with similar distinction for the groups as in Table 3.

\begin{tabular}{crrrrrrrrr}
\hline & \multicolumn{2}{c}{$\begin{array}{c}\text { Condition Homogeneous } \\
\text { (Group 4, 5, 7, 9, 10) }\end{array}$} & \multicolumn{2}{c}{$\begin{array}{c}\text { Condition Homogeneous } \\
\text { (Group 1, 2, 3, 6, 8) }\end{array}$} & \multicolumn{2}{c}{$\begin{array}{c}\text { Condition Heterogeneous } \\
\text { (All ten groups) }\end{array}$} \\
\hline Round & \multicolumn{1}{c}{ Private } & \multicolumn{1}{c}{ Carpool } & \multicolumn{1}{c}{ Shuttle } & \multicolumn{1}{c}{ Private } & Carpool & Shuttle & Private & Carpool & Shuttle \\
\hline $1-10$ & 0.13 & -0.13 & 0 & 0.24 & -0.2 & -0.04 & 0.44 & -0.21 & -0.23 \\
$11-20$ & -0.22 & 0.08 & 0.14 & 0.22 & -0.04 & -0.18 & 0.11 & -0.11 & 0 \\
$21-30$ & -0.08 & -0.34 & 0.42 & 0.1 & -0.04 & -0.06 & 0.06 & -0.01 & -0.05 \\
$31-40$ & -0.08 & 0 & 0.08 & 0.04 & -0.02 & -0.02 & 0.01 & -0.01 & 0 \\
$41-50$ & 0 & 0 & 0 & -0.02 & 0 & 0.02 & 0.01 & -0.02 & 0.01 \\
\hline Overall & -0.05 & -0.08 & 0.13 & 0.12 & -0.06 & -0.06 & 0.13 & -0.07 & -0.05 \\
\hline
\end{tabular}

* The entries for Round 1-10 aggregate changes from round 1 to 2, 2 to $3 \ldots$ up to 9 to 10 . The entries for Round 11-20 aggregate changes from round 10 to 11,11 to $12 \ldots$ up to 19 to 20 . The entries for the other three blocks are similarly aggregated as for Round 11-20. 
Table 5. Percentage frequencies of ex post best responses by block and condition, with similar distinction for the groups as in Table 3.

\begin{tabular}{cccccccccc}
\hline & \multicolumn{2}{c}{$\begin{array}{c}\text { Condition Homogeneous } \\
\text { (Group 4, 5, 7, 9, 10) }\end{array}$} & \multicolumn{2}{c}{$\begin{array}{c}\text { Condition Homogeneous } \\
\text { (Group 1, 2, 3, 6, 8) }\end{array}$} & \multicolumn{3}{c}{$\begin{array}{c}\text { Condition Heterogeneous } \\
\text { (All ten groups) }\end{array}$} \\
\hline Round & Private & Carpool & Shuttle & Private & Carpool & Shuttle & Private & Carpool & Shuttle \\
\hline $1-10$ & 82.8 & 14.6 & 2.6 & 87.4 & 0 & 12.6 & 89.3 & 10.5 & 0.2 \\
$11-20$ & 45.0 & 17.8 & 37.2 & 97.4 & 0 & 2.6 & 99.3 & 0.7 & 0 \\
$21-30$ & 23.0 & 14.6 & 62.4 & 100 & 0 & 0 & 100 & 0 & 0 \\
$31-40$ & 0 & 0 & 100 & 100 & 0 & 0 & 100 & 0 & 0 \\
$41-50$ & 0 & 0 & 100 & 100 & 0 & 0 & 98.3 & 1.7 & 0 \\
\hline Overall & 30.2 & 9.4 & 60.4 & 97.0 & 0 & 3.0 & 97.4 & 2.6 & 0.0 \\
\hline
\end{tabular}

Table 6. Percentage frequencies of route choices relative to ex post best response by block and condition, with similar distinction for the groups as in Table 3.*

\begin{tabular}{ccccccccccc}
\hline & \multicolumn{3}{c}{$\begin{array}{c}\text { Condition Homogeneous } \\
\text { (Group 4, 5, 7, 9, 10) }\end{array}$} & \multicolumn{3}{c}{$\begin{array}{c}\text { Condition Homogeneous } \\
\text { (Group 1, 2, 3, 6, 8) }\end{array}$} & \multicolumn{2}{c}{$\begin{array}{c}\text { Condition Heterogeneous } \\
\text { (All ten groups) }\end{array}$} \\
\hline Round & Lower & Equal & Higher & Lower & Equal & Higher & Lower & Equal & Higher \\
\hline $2-10$ & 0.4 & 59.1 & 40.4 & 1.8 & 58.7 & 39.6 & 1.8 & 62.7 & 35.6 \\
$11-20$ & 2.2 & 80.0 & 17.8 & 1.4 & 83.8 & 14.8 & 0.6 & 78.4 & 21.0 \\
$21-30$ & 5.4 & 73.6 & 21.0 & 0.0 & 93.2 & 6.8 & 0.0 & 88.0 & 12.0 \\
$31-40$ & 3.8 & 96.2 & 0.0 & 0.0 & 95.8 & 4.2 & 0.1 & 90.6 & 9.3 \\
$41-50$ & 2.2 & 97.8 & 0.0 & 0.0 & 96.4 & 3.6 & 0.8 & 90.1 & 9.1 \\
\hline Overall & 2.9 & 81.8 & 15.4 & 0.6 & 86.1 & 13.3 & 0.6 & 82.4 & 17.0 \\
\hline
\end{tabular}

* A choice being "lower" ("higher") means that it is ranked lower (higher) than the ex post best response; in other words, its associated equilibrium is less (more) efficient than that of the best response. Note also that the proportions in the "Lower" ("Higher") columns could be partly affected by whether there were many observations with private route (shuttle) as ex post best response - there were no lower (higher)-ranked route to choose in those observations, but they still contributed towards the base count. See Section 6.3 for more details. 
Table 7. Percentage frequencies of strategic teaching decisions by degree and condition, with similar distinction for the groups as in Table 3. The "no. of obs" entry denotes the number of observations with which strategic teaching was definable, which forms the base for the calculation of corresponding percentage frequencies.

\begin{tabular}{cccc}
\hline Degree & $\begin{array}{c}\text { Condition Homogeneous } \\
\text { (Group 4, 5, 7, 9, 10) } \\
\text { No. of Obs }=989\end{array}$ & $\begin{array}{c}\text { Condition Homogeneous } \\
\text { (Group 1, 2, 3, 6, 8) }\end{array}$ & $\begin{array}{c}\text { Condition Heterogeneous } \\
\text { (All groups) }\end{array}$ \\
\hline 0 & 62.0 & 86.3 & No. of Obs $=4899$ \\
1 & 15.3 & 5.5 & 83.0 \\
2 & 8.1 & 2.6 & 10.1 \\
3 & 4.4 & 1.4 & 3.2 \\
4 & 2.6 & 0.9 & 1.3 \\
5 & 1.8 & 0.5 & 0.8 \\
6 & 1.2 & 0.4 & 0.5 \\
7 & 0.9 & 0.4 & 0.3 \\
8 & 0.7 & 0.3 & 0.2 \\
9 & 0.6 & 0.2 & 0.1 \\
10 & 0.3 & 0.2 & 0.1 \\
11 & 0.2 & 0.1 & 0.1 \\
12 & 0.1 & 0.1 & 0.0 \\
13 & 0.1 & 0.1 & 0.0 \\
14 & 0.1 & 0.1 & 0.0 \\
\hline 15 & 1.5 & 0.8 & 0.0 \\
\hline
\end{tabular}


Figure 1. The decision screen in Condition Homogeneous.

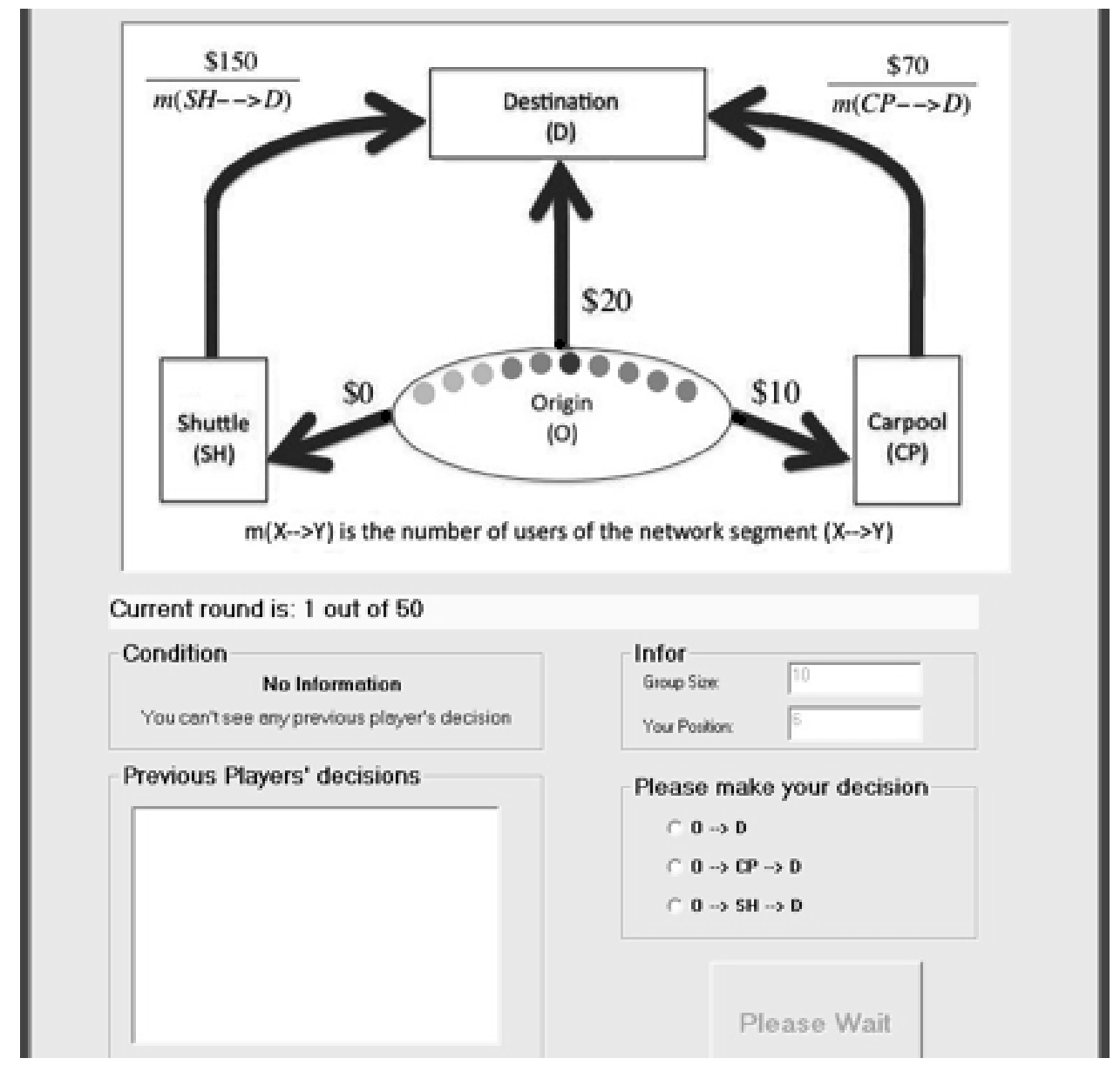


Figure 2. Route choices of the ten subjects in Group 4 of Condition Homogeneous by round and player.

Each of columns 2 to 11 registers the choices of one subject. $d$-private; $c$-carpool; $s$-shuttle

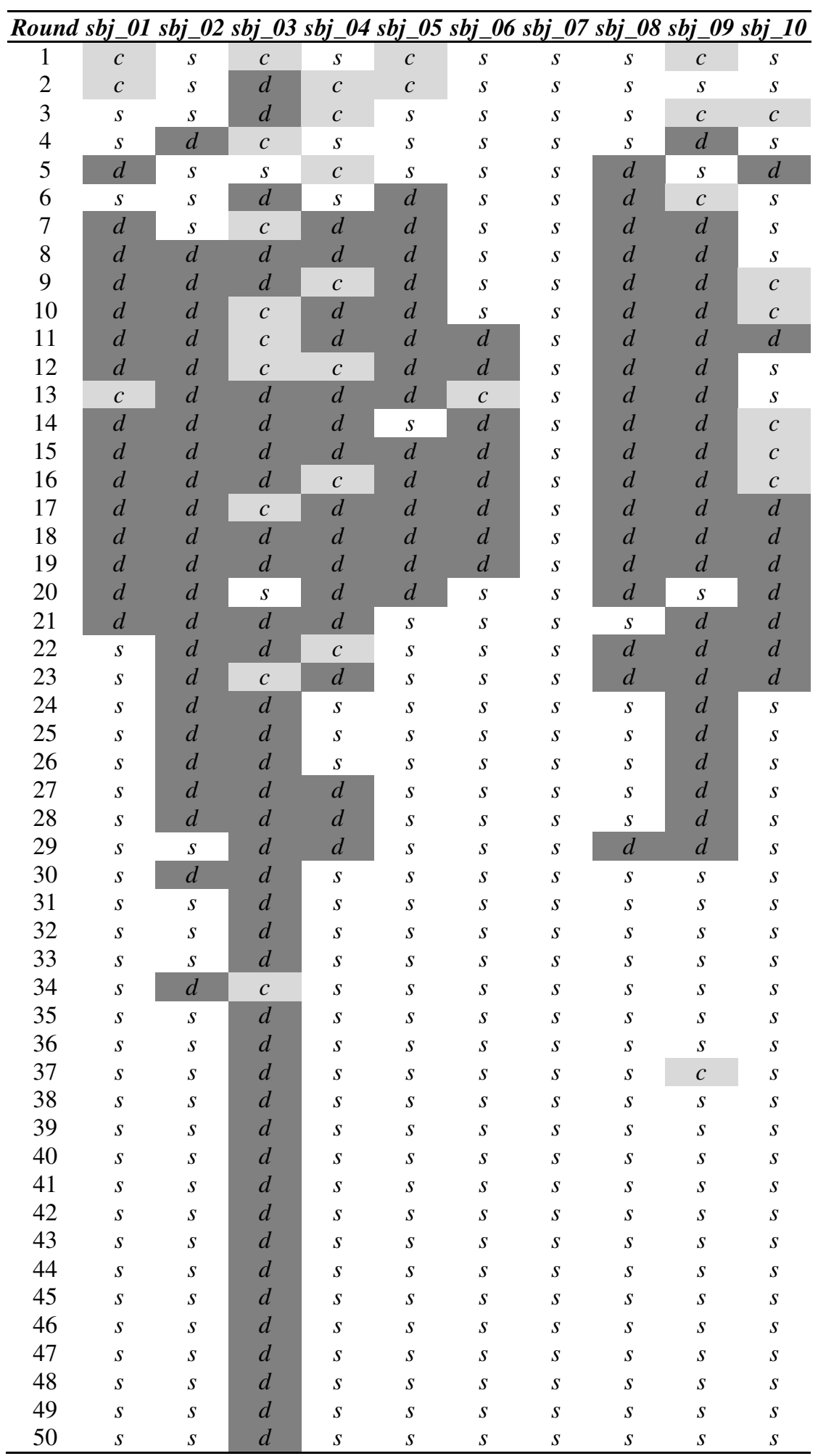


Figure 3. Number of subjects choosing each route by group and round: Condition Homogeneous.

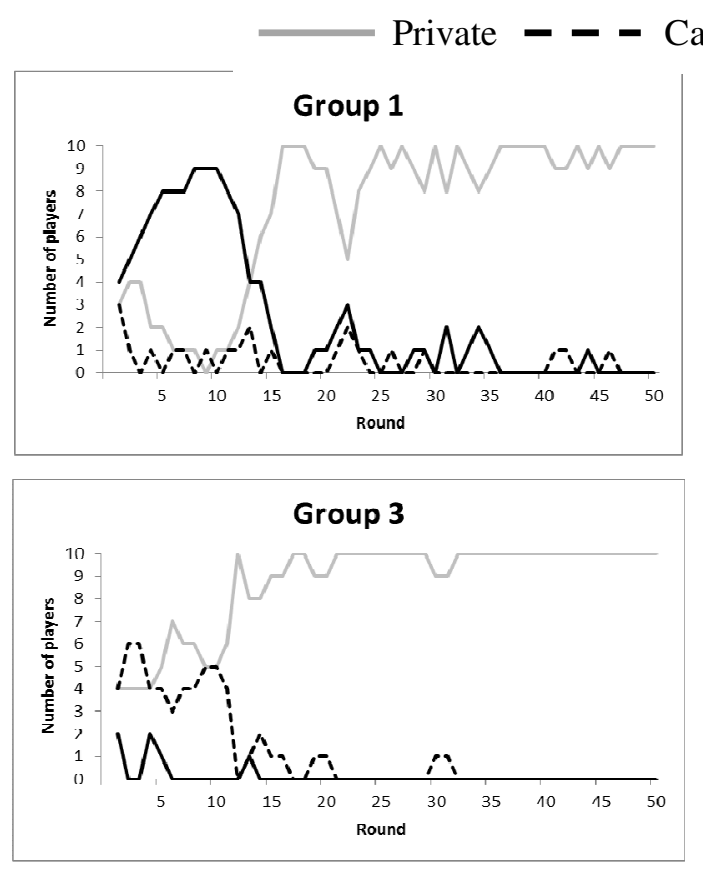

Shuttle
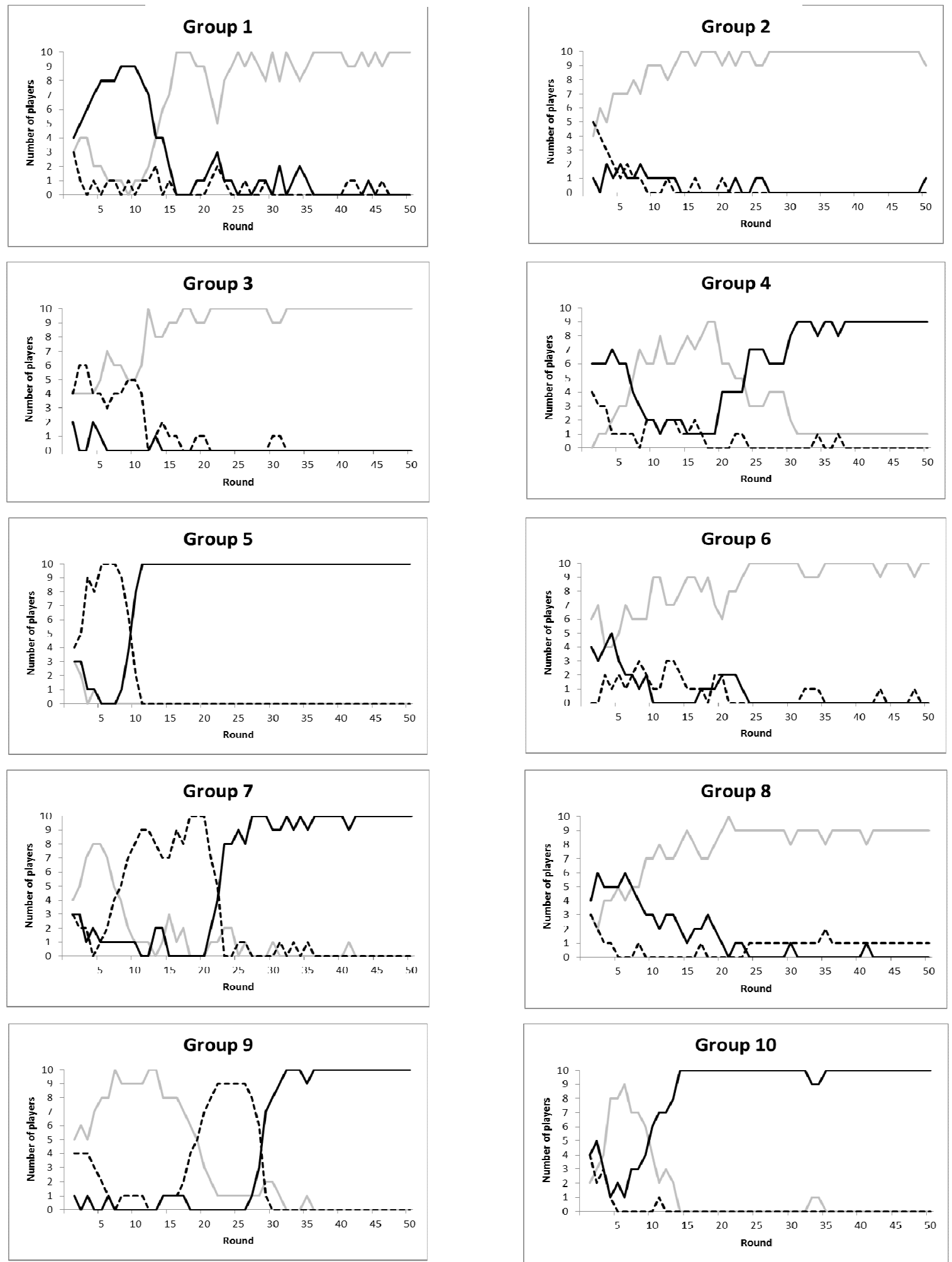
Figure 4. Number of subjects choosing each route by group and round: Condition Heterogeneous.

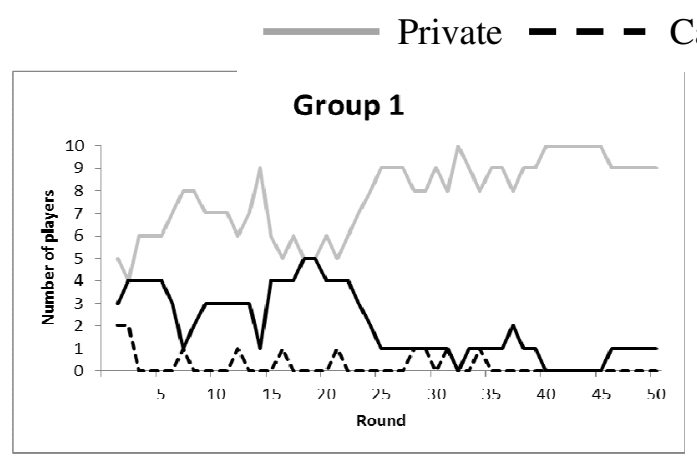

Carpool
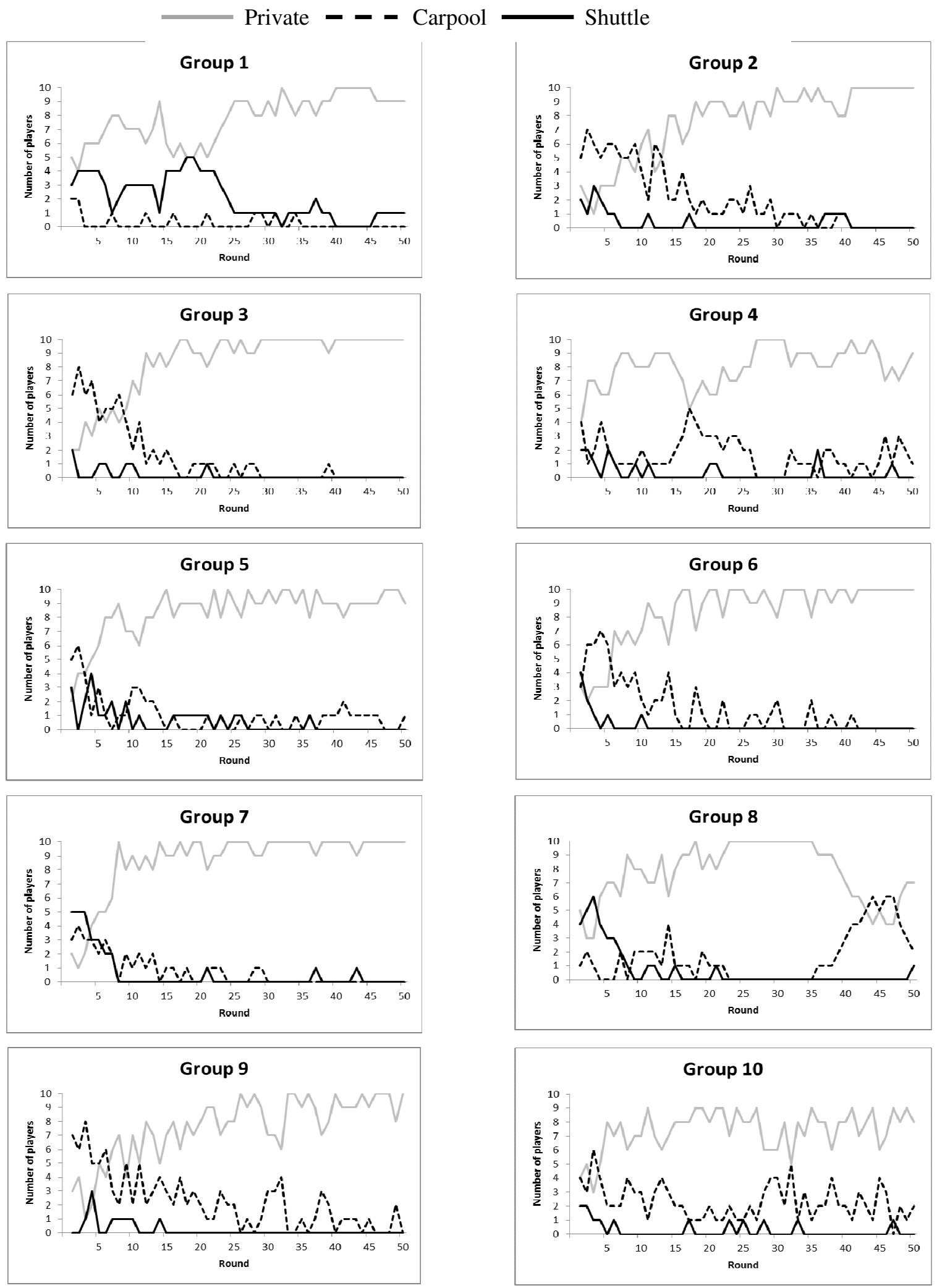
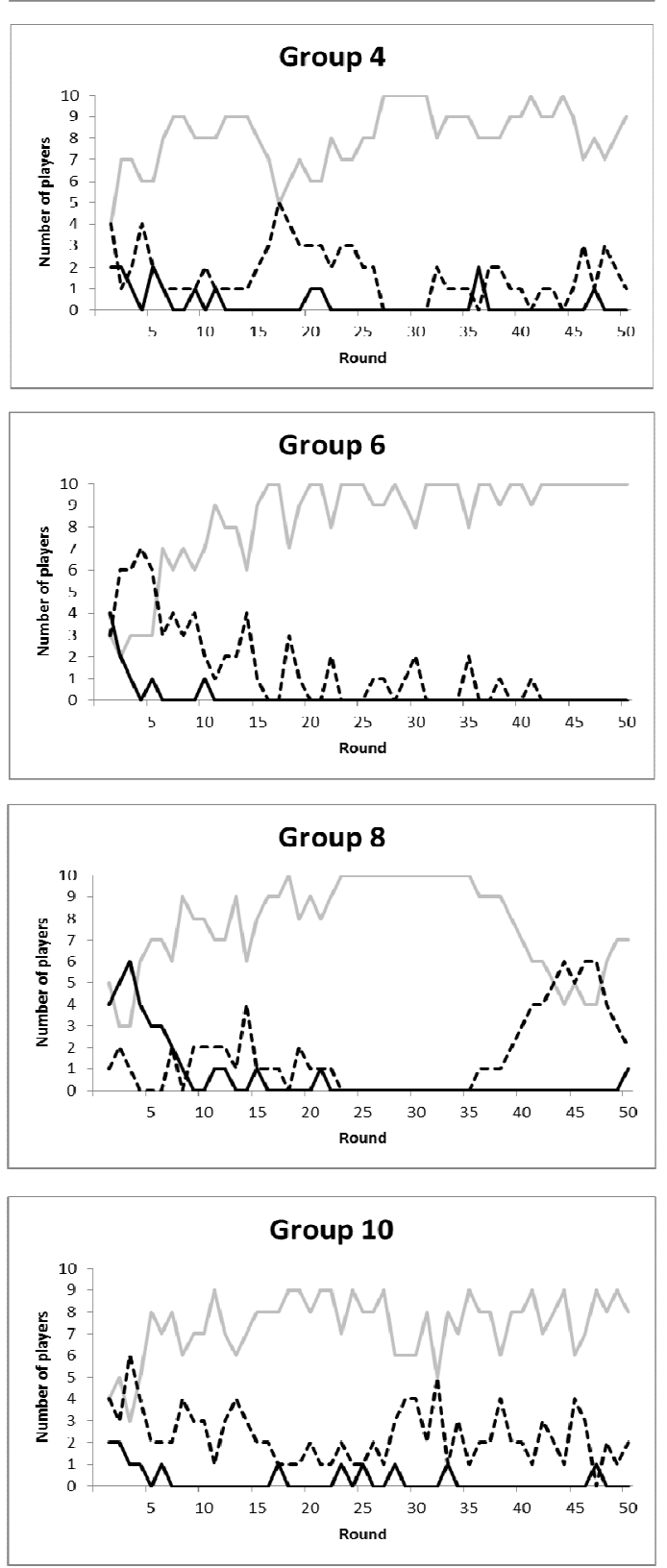
Figure 5. Percentage of subjects who switched routes by round, type of convergence (for groups in Condition Homogeneous), and condition.

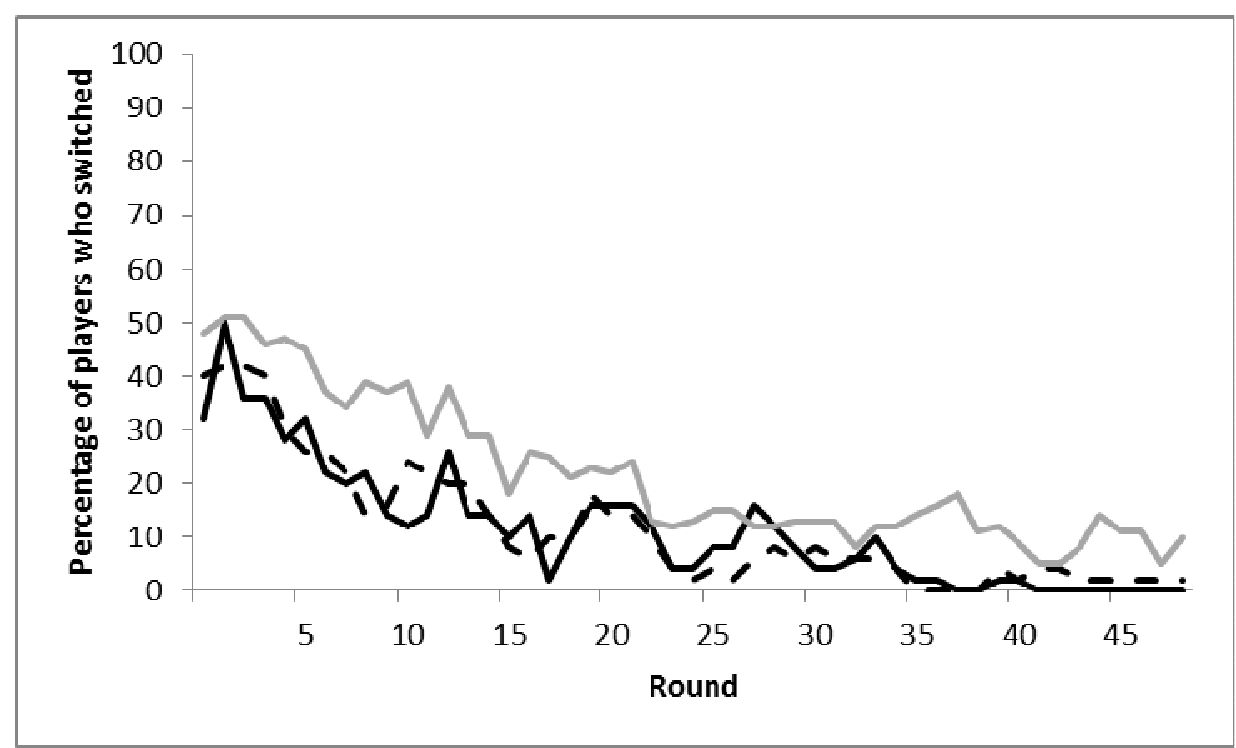

Condition Homogeneous, groups that converged towards the shuttle equilibrium

- - Condition Homogeneous, groups that converged towards the private route equilibrium Condition Heterogeneous, all groups 


\section{Appendix A: Instructions for Condition Heterogeneous \\ Instructions}

Welcome to an experiment on cost sharing. During the present experimental session, you will be asked to make a large number of decisions and so will the other participants. Your decisions, as well as the decisions of the other participants, will determine your monetary payoff according to a procedure that will be explained below.

Please read the instructions carefully. If at any time during the session you have questions, please raise your hand and one of the experimenters will come to assist you. You may refer to the instructions during any time in the session.

Please note that from now on all communication between the participants is prohibited. If the participants communicate with one another in any shape or form, the session will be terminated. Please note, too, that the experiment is self-paced. Therefore, you may anticipate short delays while other participants in your group determine and then type in their decisions.

\section{Description of the Task}

Consider yourself to be one of $\mathbf{1 0}$ commuters, each owning a car. In this experiment, you will be asked to participate in a task that simulates the decisions commuters often face in choosing among alternative modes of transportation between a given origin (say, a convention center) and a given destination (say, a restaurant for the conference dinner).The game consists of many identical rounds. Each round is described as follows.

The commuters (hereafter called "players") will be asked to make their decisions one by one following an order which is pre-determined randomly by the computer. Each player has to choose one of three alternatives:

- $\mathbf{O} \rightarrow \mathbf{D}$ : Drive your car by yourself from the origin (denoted by the letter $\mathbf{0}$ ) to the destination (denoted by the letter D). The cost of traveling by yourself in your own car (called the OD cost) is randomly drawn from the interval [16,24], i.e., it can take any value from 16 to 24 with equal probabilities. The other players' OD costs are also randomly drawn from the interval [16,24]. At the beginning of each round, every player's OD cost is independently determined in this way, and each player is informed of her own cost only. You don't know the exact value of other players' OD cost and they don't know yours as well. The OD costs are re-determined by random draws from the interval [16,24] at the beginning of each round, and so may be the same or different from round to round. 
- $\mathbf{O} \rightarrow \mathbf{C P} \rightarrow \mathbf{D}$ : Drive your car from the origin to a nearby carpool lot, park your car, and then travel with the carpool to your destination. You pay $\$ \mathbf{1 0 . 0 0}$ for parking your car. In addition, the cost charged by the carpool is $\mathbf{\$ 7 0 . 0 0}$, which is divided equally by the number of players who choose the carpool. The capacity of the carpool is 10 . In other words, you share equally the cost of public transportation. Thus, your travel cost from $\mathbf{O}$ to $\mathbf{D}$ through route $\mathbf{O} \rightarrow \mathbf{C P} \rightarrow \mathbf{D}$ is

$$
\$ 10+\frac{\$ 70}{\mathbf{m}(\mathbf{C P} \rightarrow \mathrm{D})}
$$

where $\mathrm{m}(\mathbf{C P} \rightarrow \mathbf{D})$ is the number of players who share the carpool.

- $\mathbf{O} \rightarrow \mathbf{S H} \rightarrow \mathbf{D}$ : Drive your car from the origin to a shuttle station, park your car, and travel with the shuttle to your destination. There is no charge for parking your car in the shuttle station. The total cost of the shuttle is $\mathbf{\$ 1 5 0 . 0 0}$, which is divided equally by the number of players riding the shuttle. The capacity of the shuttle is 10. Thus, your travel cost from $\mathbf{O}$ to $\mathbf{D}$ through route $\mathbf{O} \rightarrow \mathbf{S H} \rightarrow \mathbf{D}$ is

$$
\frac{\$ 150}{m(S H \rightarrow D)}
$$

where $\mathrm{m}(\mathbf{S H} \rightarrow \mathbf{D})$ is the number of players who share the ride by the shuttle.

This is a sequential game in which players make their decisions one by one. Each player will be assigned a different position in the sequence (from 1 to 10) at the beginning of each round. Then, each player will be asked to type in her decision when it is her turn to play. Moreover, when a player makes her choice, she has no information about the choices of the players who preceded her in the sequence. The game will be iterated for 50 rounds; they only differ from each other in the assignment of the positions.

\section{$\underline{\text { Experiment Procedure }}$}

At the beginning of each round, each of the 10 players will be presented with the following diagram. A message window will pop up to indicate the round number and your position in the sequence of choosing the mode of transportation. The position of each player will be randomly assigned by the computer, and in general will change from one round to another. During the experiment, each player will be assigned any given position the same number of times. 


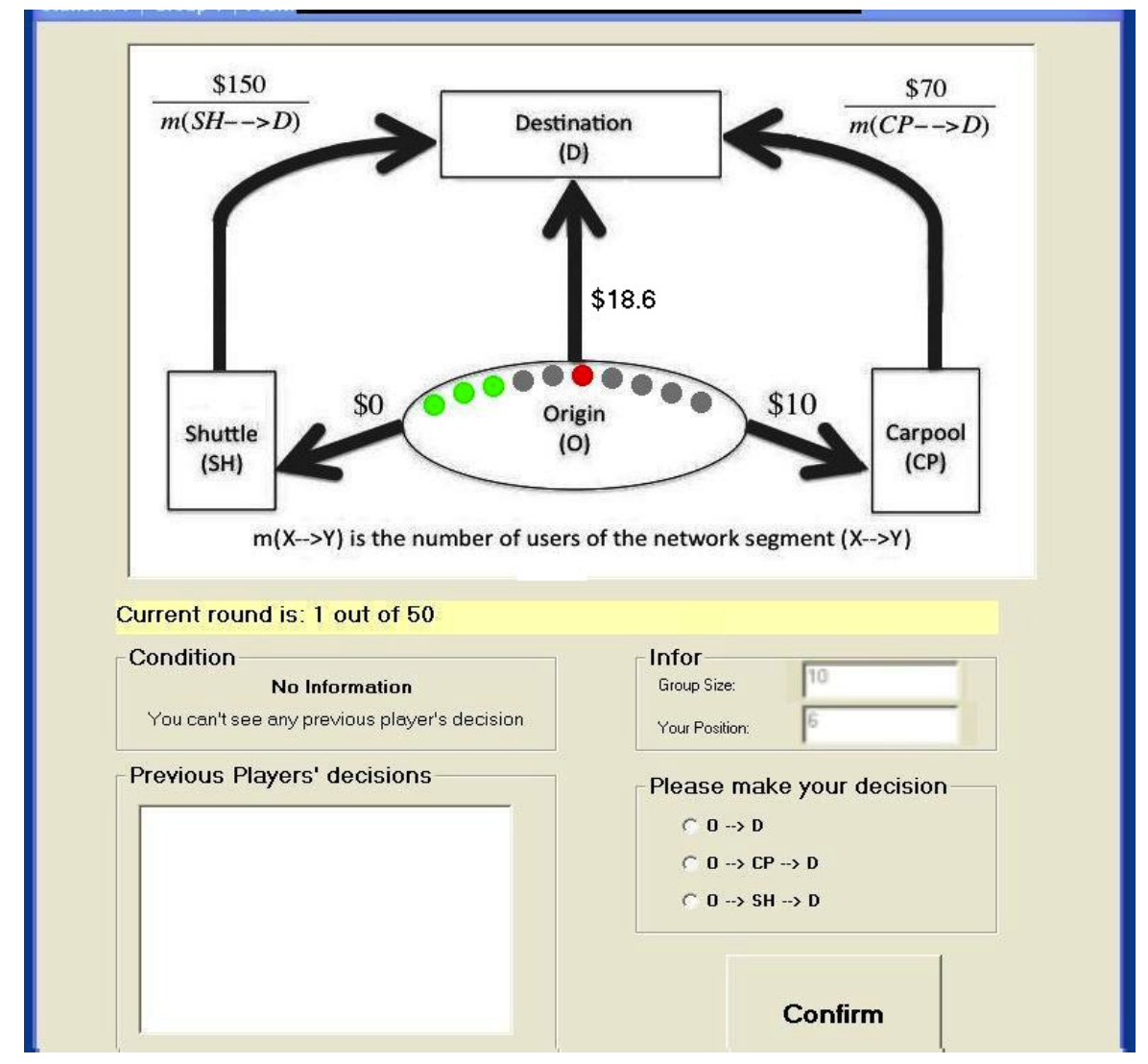

Please study the main screen above. The upper part of the screen is a sketch map of the traffic system. There are 10 cars parked in the origin; each is exhibited as a solid circle. When it is her turn to play, each player can either drive to the destination $\mathbf{D}$ (up arrow) and pay her private OD cost, (for example as shown in the diagram, the $\mathbf{O D}$ cost is 18.6 in this round), drive to the carpool station $\mathbf{C P}$ (right arrow), park her car, and then take the carpool for a total cost of $\$ 10+\$ 70 / \mathrm{m}(\mathrm{CP} \rightarrow \mathrm{D})$, or drive to the shuttle station SH (left arrow), park her car (for free), and then take the shuttle for a total cost of $\$ 150 / \mathbf{m}(\mathbf{S H} \rightarrow \mathbf{D})$. In the diagram, the number shown on the upper arrow is the $\mathbf{O D}$ cost for the current round. Each player has her own OD cost. In general, this cost will change across different rounds.

On the screen, grey solid circles represent players who are waiting to be called to make their decision. Green solid circles represent players who have already made their decisions. And the single red solid circle represents each player's current position. After each player makes her decision, her red solid circle will turn to green. The middle of the screen shows a status bar (in yellow) that indicates the progress of the experiment. It tells you how many rounds have been completed and the total number of rounds. 
In the lower part of the screen there are two tables. The top table on the right, labeled infor, shows the group size (which is 10) and your position in the sequence. The bottom table is where you make your decision. Use the mouse to choose one of the three options. If you change your mind, please choose another option. Your decision will not register until you press the Confirm button (lower right). The left part of the lower screen, labeled Condition, tells you if you have any information about the decisions of other players. Here it says No Information, meaning that the decisions of the other players are not displayed.

Once all the ten players type in their decisions and confirm them, a new screen will pop up to display the outcome of the round. This screen shows the number of players who have chosen each of three routes, the cost associated with each decision, your decision, your cost for the round, and your payoff in points. See an example in screen below.

Please study the outcome screen below.

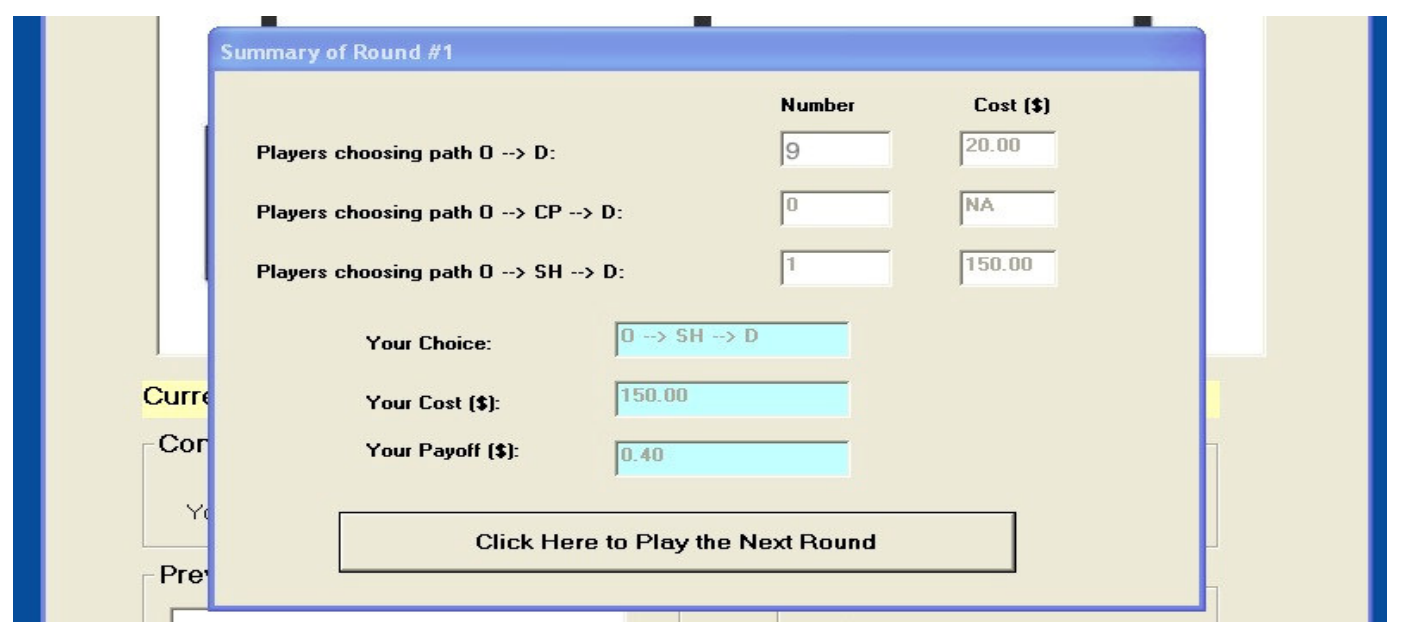

After clicking the "Click here to play the next round" at the bottom of this screen, a window will pop up instructing the player to wait for all the players to review the outcome of the round. Then, a new round will start. The game will be repeated in this way for 50 rounds. The only difference from one round to another is in the order of the players that are assigned randomly by the computer.

\section{How will you be paid?}

When all the 50 rounds have been completed, the computer will choose randomly $\mathbf{5}$ payoff rounds for each player. Payoff of each round will then be converted into US dollars at the rate: 


$$
\text { payoff }=\frac{\$ 60}{\operatorname{cost}}
$$

For example, if your cost in a chosen round is $\$ 15$, your payoff in that round will be $\$(60 / 15)=\$ 4$. Your final payoff will be the sum of the payoffs from the 5 randomly selected rounds. In addition, you will be paid $\$ \mathbf{5 . 0 0}$ for your participation in the experiment. The experimenter will come up to your cubicle and write your total payoff on your receipt. You'll complete the receipt, sign it up, and then be paid cash by one of the experimenters.

Please place the instructions on the table in front of you to indicate that you have completed reading them. The experiment will begin shortly. Please remember that no communication is allowed during the experiment. If you encounter any difficulties please raise your hand and you will be responded to by the experimenter.

Thank you! 


\section{Appendix B: Further Analysis on Switching Frequencies}

The following table lists the group average of the number of switches in different directions, by condition and the type of equilibrium convergence:

\begin{tabular}{|c|c|c|c|c|c|c|c|c|c|}
\hline \multirow[b]{2}{*}{ Condition } & \multirow[b]{2}{*}{ Convergence } & \multicolumn{4}{|c|}{ Switches towards a more efficient equilibrium } & \multicolumn{4}{|c|}{ Switches towards a less efficient equilibrium } \\
\hline & & p to $c$ & p to $s$ & c to $s$ & Total & c to $p$ & $s$ to $p$ & $s$ to $c$ & Total \\
\hline Homogeneous & Private & 12 & 10.8 & 2.8 & 25.6 & 15.6 & 12.8 & 3.6 & 32 \\
\hline Homogeneous & Shuttle & 11.4 & 10.4 & 9.6 & 31.4 & 11.4 & 7.8 & 5.8 & 25 \\
\hline Heterogeneous & Private (mostly) & 38.2 & 7.9 & 4.3 & 50.4 & 41.9 & 10.1 & 4.6 & 56.6 \\
\hline
\end{tabular}

Note: The "Convergence" entry indicates the type of equilibrium which the groups converged towards, where "Private" denotes the inefficient equilibrium with all players choosing Private and "Shuttle" denotes the efficient equilibrium with all players choosing Shuttle. For the labeling of the switches, $d$ denotes private, $c$ denotes carpool, $s$ denotes shuttle.

We further analyze whether the total number of switches towards a more versus less efficient equilibrium (i.e., columns 6 and 10) differs by the type of equilibrium convergence and the experimental condition. Paired $t$-tests with group as the unit of analysis show that:

1. Among the groups in Condition Homogeneous that converged towards the inefficient equilibrium, as well as among the groups in Condition Heterogeneous, there were significantly more switches towards a less efficient than towards a more efficient equilibrium $(p<0.01$ in both $t$ tests). This confirms the intuition that in those groups, switches by subjects brought about convergence to inefficiency.

2. Among the groups in Condition Homogeneous that converged towards the efficient equilibrium, there is no significant difference between the switches towards a less efficient and towards a more efficient equilibrium $(p=0.07)$. As discussed in the main text, switches occurred more frequently in the early part of the session. This result is consistent with the high instability in the early rounds among groups that eventually converged towards the efficient equilibrium: there could be a tug of war between the tendency towards the efficient and inefficient equilibria, until a tip of the balance in the middle of the session. 
Further $t$-tests, however, show that the frequency of neither type of switches differs significantly between groups with different types of convergence in Condition Homogeneous ( $p>0.3$ for both types of switches). That is, equilibrium convergence was characterized more by the difference between the types of switches than by the absolute number of the switches. 